\title{
NESTED SHELLS REVEAL THE REJUVENATION OF THE ORION-ERIDANUS SUPERBUBBLE
}

\author{
Bram B. Ochsendorf ${ }^{1}$, Anthony G. A. Brown ${ }^{1}$, John Bally ${ }^{2}$, and Alexander G. G. M. Tielens ${ }^{1}$ \\ ${ }^{1}$ Leiden Observatory, Leiden University, P.O. Box 9513, NL-2300 RA, The Netherlands; ochsendorf@strw.leidenuniv.nl \\ ${ }^{2}$ CASA, APS, UCB389, University of Colorado, Boulder, CO 80389, USA \\ Received 2015 January 5; accepted 2015 June 5; published 2015 July 27
}

\begin{abstract}
The Orion-Eridanus superbubble is the prototypical superbubble owing to its proximity and evolutionary state. Here we provide a synthesis of recent observational data from WISE and Planck with archival data, allowing us to draw a new and more complete picture on the history and evolution of the Orion-Eridanus region. We discuss the general morphological structures and observational characteristics of the superbubble and derive quantitative properties of the gas and dust inside Barnard's Loop. We reveal that Barnard's Loop is a complete bubble structure that, together with the $\lambda$ Ori region and other smaller-scale bubbles, expands within the Orion-Eridanus superbubble. We argue that the Orion-Eridanus superbubble is larger and more complex than previously thought, and that it can be viewed as a series of nested shells, superimposed along the line of sight. During the lifetime of the superbubble, H II region champagne flows and thermal evaporation of embedded clouds continuously massload the superbubble interior, while winds or supernovae from the Orion OB association rejuvenate the superbubble by sweeping up the material from the interior cavities in an episodic fashion, possibly triggering the formation of new stars that form shells of their own. The steady supply of material into the superbubble cavity implies that dust processing from interior supernova remnants is more efficient than previously thought. The cycle of mass loading, interior cleansing, and star formation repeats until the molecular reservoir is depleted or the clouds have been disrupted. While the nested shells come and go, the superbubble remains for tens of millions of years.
\end{abstract}

Key words: H II regions - ISM: bubbles - ISM: clouds - ISM: kinematics and dynamics - stars: formation

\section{INTRODUCTION}

The Orion-Eridanus superbubble is a nearby $(\sim 400 \mathrm{pc})$ expanding structure that is thought to span $20^{\circ} \times 45^{\circ}$ on the sky (Reynolds \& Ogden 1979; Bally 2008; Pon et al. 2014b). Because of its proximity and evolutionary stage, it is traced at a multitude of wavelengths (Heiles et al. 1999), serving as a benchmark to the study of superbubbles. The expansion of the bubble is most likely connected to the combined effects of ionizing UV radiation, stellar winds, and a sequence of supernova (SN) explosions from the Orion OB association, Orion OB1 (Blaauw 1964; Brown et al. 1994).

The line-of-sight expansion of the Orion-Eridanus superbubble is previously estimated at $\sim 15 \mathrm{~km} \mathrm{~s}^{-1}$ through line splitting of $\mathrm{H} \alpha$, which also revealed a total ionized gas mass of $8 \times 10^{4} M_{\odot}$ and a kinetic energy of $E_{\text {kin }}>1.9 \times 10^{50} \mathrm{erg}$ (Reynolds \& Ogden 1979). Large-scale spectroscopic mapping of the entire Orion-Eridanus region in $\mathrm{H}$ I reported a larger expansion velocity of the superbubble of $\sim 40 \mathrm{~km} \mathrm{~s}^{-1}$ with a mass of $2.5 \times 10^{5} M_{\odot}$, containing a kinetic energy of $3.7 \times 10^{51}$ erg (Brown et al. 1995), which is shown to be consistent with the integrated mechanical luminosity exerted by Orion OB1 $\left(\sim 10^{52} \mathrm{erg}\right.$; Brown et al. 1995). The expansion of the superbubble is also traced through high-velocity (HV) and intermediate-velocity (IV) gas in several lines of sight toward Orion (Cowie et al. 1979; Welty et al. 2002). Soft X-rays emanate from the million-degree plasma in the interior of the superbubble (Burrows et al. 1993; Snowden et al. 1995).

Here we present observations of recent all-sky surveys with WISE and Planck and combine them with existing sky surveys to provide a new and more complete insight into the history and evolution of the Orion-Eridanus region. In particular, we will reveal that Barnard's Loop is part of a complete bubble structure, separate from the Orion-Eridanus superbubble, that sweeps up the mass-loaded interior of the preexisting superbubble. It is argued that the Orion-Eridanus superbubble is larger and more complex than previously thought, and that the entire morphological appearance of the superbubble can be viewed as a series of nested shells, superimposed along the line of sight. The shells originate from explosive feedback from Orion OB1 that accelerates, sweeps up, and compresses the superbubble interior plasmas in an episodic fashion to form nested shells within the Orion-Eridanus superbubble. We explore the origin of the shells, their relation with the subgroups of Orion OB1, and their impact on the molecular clouds and star formation efficiency within Orion. We discuss our findings in terms of the long-term evolution of the superbubble. We present the obervations in Section 2 and our results in Section 3. We discuss our findings in Section 4 and summarize in Section 5.

\section{OBSERVATIONS}

We made use of the all-sky surveys from Planck (Planck Collaboration et al. 2014c), the Wide-field Infrared Explorer (WISE; Wright et al. 2010), the Leiden/Argentina/Bonn survey of Galactic H I (LAB; Kalberla et al. 2005), the ROSAT soft X-ray background (Snowden et al. 1997), and an all-sky $\mathrm{H} \alpha$ map that combines several large-scale surveys (Finkbeiner 2003), including the Southern H-alpha Sky Survey Atlas (Gaustad et al. 2001), the Virginia Tech Spectral-line Survey

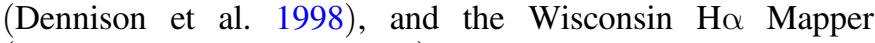
(WHAM; Haffner et al. 2003).

\section{RESULTS}

Figure 1 reveals the large-scale structure of the OrionEridanus superbubble. In $\mathrm{H} \alpha$, the region exhibits various 

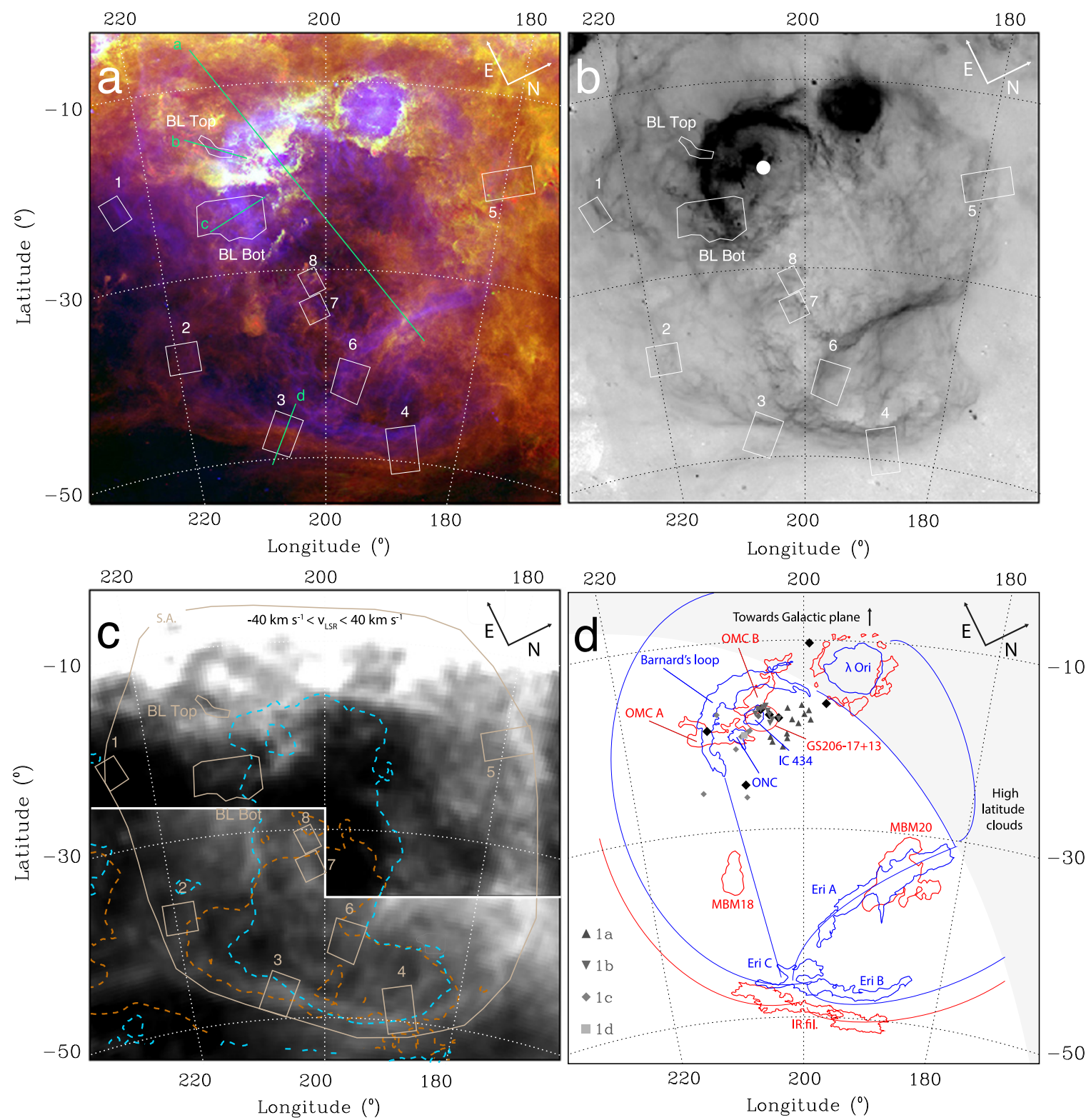

Figure 1. (a) Three-color image of the Orion-Eridanus superbubble. $\mathrm{H} \alpha$ (blue) reveals ionized gas, surrounded by a layer of polycyclic aromatic hydrocarbons or stochastically heated small grains, traced by the WISE $12 \mu \mathrm{m}$ band (green). Red is Planck $353 \mathrm{GHz}$ that probes columns of cold (20 K) dust. The polycyclic aromatic hydrocarbons/small grains trace the UV-illuminated edges of the clouds containing cold dust, resulting in that red and green blend to form yellow. To the northeast (top part the image) lies the Galactic plane, while to the northwest (right part of the image) lie high-latitude clouds (e.g., Schlafly et al. 2014). The numbered boxes define the apertures discussed in Section 3.2, while the light-green labeled solid lines mark the positions of cross-cuts used in Figure 2. (b) Same region as panel (a), but only showing the $\mathrm{H} \alpha$ emission using a different stretch to accentuate the faint shell structure that envelops the Orion region in $\mathrm{H} \alpha$. The white dot reveals the fluxweighted center of the $\mathrm{H} \alpha$ bubble (Reynolds \& Ogden 1979). (c) H I emission from the LAB survey, integrated in velocity space along the full extent of the OrionEridanus superbubble, $-40 \mathrm{~km} \mathrm{~s}^{-1}<v_{\mathrm{LSR}}<40 \mathrm{~km} \mathrm{~s}^{-1}$ (Brown et al. 1995). Overlayed are contours of ROSAT $0.25 \mathrm{keV}$ (orange dashed) and ROSAT $0.75 \mathrm{keV}$ diffuse X-rays (light blue dashed). The $0.75 \mathrm{keV}$ emission projected within Barnard's Loop originates from bright sources within the ONC and IC 434 regions, but it is blended with the more diffuse emission from the superbubble because of smoothing of the ROSAT maps. Below the white solid line the image has a different scaling

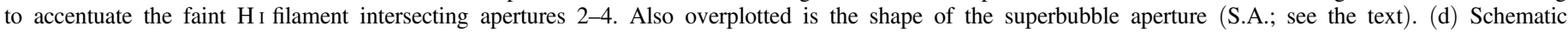
representation of the region. $\mathrm{H} \alpha$ gas structures are shown in blue lines/contours, while dust structures are plotted in red. The solid lines trace faint filaments of the region to guide the eye in panels (a) and (b). The stars that form the well-known constellation of Orion are also plotted to appreciate the immense size of the region (black diamonds). Members of the different subgroups of the Orion OB association (Blaauw 1964; Brown et al. 1994) with spectral type B2 or earlier are also shown in different gray symbols shown by the legend. See the text for the explanation of the different abbreviations.

filamentary structures, including Barnard's Loop (Barnard 1894) and the Eridanus filaments (e.g., Pon et al. 2014a). Projected on top of Barnard's Loop are the Orion A and Orion B molecular clouds (OMC A and OMC B), the Orion Nebula Cluster (ONC) associated with the Orion Nebula, and the IC 434 emission nebula that is characterized by a champagne flow of ionized gas (Tenorio-Tagle 1979;
Section 3.3.4). Another prominent feature is the $\lambda$ Orionis bubble, a $10^{\circ}$ circular $\mathrm{H}$ II region, encapsulated by a swept-up shell of gas and dust. Below we refer to different parts of the superbubble in R.A./decl. space (see the orientation in Figure 1).

Figure 2 shows cross-cuts through the Orion-Eridanus superbubble, revealing the gas and dust structures of the 

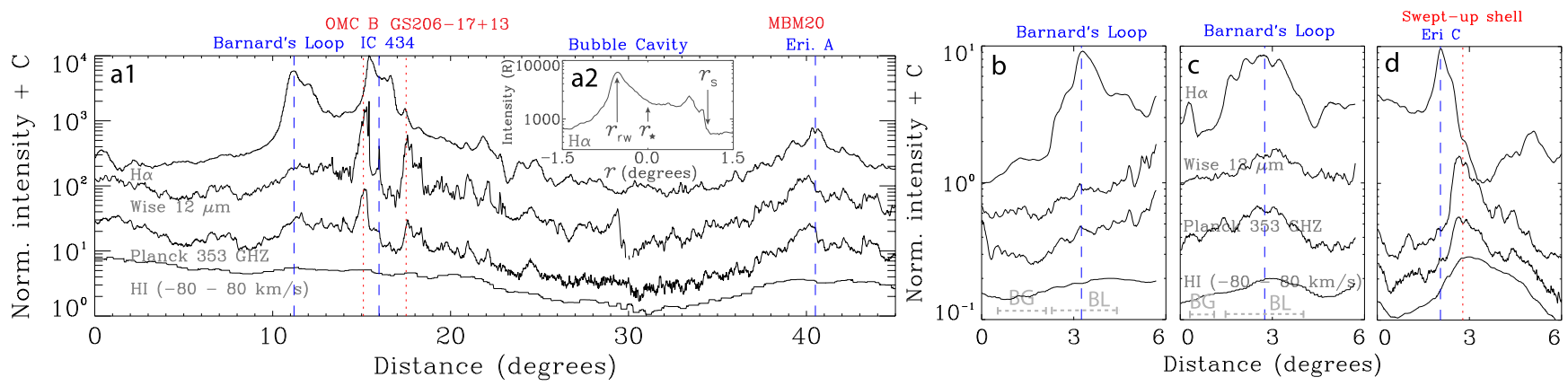

Figure 2. Profiles of cross-cuts defined in Figure 1(a). Cuts start at the labeled side in Figure 1a. (a1) Along the cut from left to right: Barnard's loop; the Orion B molecular cloud (OMC B) and the IC 434 emission nebula; the GS206-17+13 shell; the excavated bubble interior; and the MBM 20 high-latitude cloud, overlapping the Eri A H $\alpha$ filament. The dashed vertical lines highlight several prominent features seen in the gas (blue) and in dust (red). (a2) Inset of the IC 434 emission nebula $\mathrm{H} \alpha$ profile, plotted as intensity (in units of Rayleigh) as a function of distance from the ionizing star $r$ (in degrees). The emission profile exhibits the structure of a champagne flow of ionized gas (Tenorio-Tagle 1979). We have marked the location of the ionizing star, $\sigma$ Ori $\left(r_{\star}\right)$, the shock front $\left(r_{\mathrm{s}}\right)$, and the rarefaction wave $\left(r_{\mathrm{rw}}\right)$. See Section 3.3.4 for a full description of the champagne flow structure. (b) and (c) Cuts through Barnards's loop (BL top and $\mathrm{BL}_{\text {bot }}$; Heiles et al. 2000), revealing a single peak most prominently seen in $\mathrm{H} \alpha$. The dashed lines on the bottom define the bins where the mean optical depth at $353 \mathrm{GHz}$, $\tau_{353, \mathrm{obs}}$, from the Planck dust model R1.20 (Planck Collaboration et al. 2014a) is measured (see the text): "BL" is the bin for Barnard's Loop, "BG" is the bin for the background determination. (d) Cut through the western edge of the superbubble, coincident with the Eri $\mathrm{C}$ filament. Here the gas and dust reveal a stratified structure, where the ionized gas peaks inside the dust shell.

superbubble and its interior components. Note that Barnard's Loop peaks dramatically in the ionized gas, while in the dust and neutral hydrogen it is only seen as a modest increase in the Planck, WISE, and LAB channels (Figures 2(a)-(c)). At this point, we find no evidence that a dense shell encapsulates the ionized portion of Barnard's Loop, whose presence was predicted by Cowie et al. (1979) (Section 4). In contrast, the cross-cut shown in Figure 2(d) that traces the western bubble shell at high latitudes reveals a stratified structure, where a dust/ $\mathrm{H}$ I shell is clearly observed to encapsulate the Eridanus C filament. The absence of an $\mathrm{H}$ i shell associated with Barnard's Loop is surprising and encouraged us to investigate the gas and dust content of Barnard's Loop and the Orion-Eridanus superbubble in more detail; our findings are presented in the next sections.

\subsection{Dust in Barnard's Loop}

\subsubsection{Optical Depth}

Heiles et al. (2000) defined two small regions of Barnard's Loop that are clear of any dense molecular clouds along the line of sight; we use the same apertures in the following analysis ("BL $\mathrm{L}_{\text {top }}$ " and "BL $\mathrm{Bot}_{\text {bot }}$; Figure 1) to characterize the dust contained in Barnard's Loop. The distance toward Barnard's Loop is uncertain; here we assume that Barnard's Loop lies at the distance of the Orion Nebula ( $400 \mathrm{pc}$; Menten et al. 2007; Sandstrom et al. 2007). Electron densities inside Barnard's Loop are estimated at $n_{\mathrm{e}}=2.0 \mathrm{~cm}^{-3}$ (Heiles et al. 2000) and at $n_{\mathrm{e}}=3.2 \mathrm{~cm}^{-3}$ from the surface brightness of the $\mathrm{H} \beta$ line (O'Dell et al. 2011). A similar analysis of the $\mathrm{H} \alpha$ surface brightness here leads to $n_{\mathrm{e}}=3.4 \mathrm{~cm}^{-3}$, after correcting for limb brightening (a factor of 7; O'Dell et al. 2011) of a constant density shell with an apparent radial thickness of $1^{\circ}$, which amounts to $7 \mathrm{pc}$ at the distance of $400 \mathrm{pc}$.

Using $n_{\mathrm{e}}=3.4 \mathrm{~cm}^{-3}$ and a radial thickness of $7 \mathrm{pc}$, adopting a normal gas-to-dust ratio of $N_{\mathrm{H}} / A_{\mathrm{V}}=1.9 \times 10^{21} \mathrm{~cm}^{-2} \mathrm{mag}^{-1}$ (Bohlin et al. 1978), where $N_{\mathrm{H}}$ is the column density of hydrogen and $A_{\mathrm{V}}$ the visual extinction, and a UV-to-visual dust absorption ratio of 1.8 (Tielens \& Hollenbach 1985), the radial dust optical depth for UV photons with energies between 6 and $13.6 \mathrm{eV}$ is $\tau_{\mathrm{UV}}=0.07$.
Through a modified-blackbody fit to the Planck data, the Planck R1.20 dust model provides all-sky maps of dust optical depth (at $353 \mathrm{GHz}$ ), temperature, spectral index, and the total dust emission integrated over frequency. We obtain an observed optical depth of Barnard's Loop at $353 \mathrm{GHz}$, $\tau_{353, \text { obs }}$, by correcting the value of the optical depth from the Planck model at the location of Barnard's Loop for a background value. For this method we use the bins defined in Figures 2(b) and (c): as Barnard's Loop is not encapsulated by a dense shell (Figure 2 and Section 3.2), we determine the background in a region just outside of the ionized portion of Barnard's Loop (which is significant: $~ 60 \%$ of the total value for both regions). In this way, we isolate the dust grains contained within the ionized part of Barnard's Loop. Then, the observed optical depths are $\tau_{353, \mathrm{obs}}=9.0 \times 10^{-6}$ (for $\mathrm{BL}_{\text {top }}$ ) and $\tau_{353, \text { obs }}=5.1 \times 10^{-6}\left(\mathrm{BL}_{\mathrm{bot}}\right)$. We can compare this to the expected optical depth, $\tau_{353, \mathrm{cal}}$, from the density $\left(3.4 \mathrm{~cm}^{-3}\right)$, estimated line-of-sight depth ( $45 \mathrm{pc}$ for a $1^{\circ}$ thickness), and the opacity at $353 \mathrm{GHz}\left(\sigma_{\nu}=7.9 \times 10^{-27} \mathrm{~cm}^{2} \mathrm{H}^{-1}\right)$ appropriate for the diffuse interstellar medium (ISM; Planck Collaboration et al. 2014a). We calculate $\tau_{353, \text { cal }}=4.0 \times 10^{-6}$, in close agreement with $\tau_{353, \text { obs }}$, which implies that dust mixed with the ionized shell of Barnard's Loop is similar to dust seen in the diffuse ISM.

\subsubsection{Temperature}

Heiles et al. (2000) concluded that grain temperatures in Barnard's Loop are higher than in $\mathrm{H}$ I regions and proposed that $\operatorname{Ly} \alpha$ photons might contribute to the heating of the dust. However, direct heating from the radiation field of Orion OB1 was not considered in their study. We derive an intensity of $G_{0}=14$ reaching Barnard's Loop in units of the Habing field (Habing 1968), taking into account the radiation from the seven most massive stars in Orion OB1 $(\theta$ Ori $\mathrm{C}, \delta$ Ori, $\iota$ Ori, $\theta$ Ori A, $\sigma$ Ori, $\epsilon$ Ori, $\zeta$ Ori), using stellar parameters from Martins et al. (2005). However, O'Dell (2001) shows that radiation from the Orion Nebula ( $\theta$ Ori $\mathrm{C}$ and $\theta$ Ori A) might not reach Barnard's Loop, as the nebula is optically thick to ionizing radiation in all directions, except possibly to the southwest. This would decrease $G_{0}$ to 11 . 
The observed dust temperatures from the Planck model in $\mathrm{BL}_{\text {top }}$ and $\mathrm{BL}_{\text {bot }}$ are $T_{\mathrm{d}}=18.7 \mathrm{~K}$ and $T_{\mathrm{d}}=20.5 \mathrm{~K}$, respectively. These values are consistent with temperatures found by Heiles et al. (2000), $T_{\mathrm{d}}=19.6 \mathrm{~K}$ and $T_{\mathrm{d}}=20.3 \mathrm{~K}$ for $\mathrm{BL}_{\text {top }}$ and $\mathrm{BL}_{\text {bot }}$, respectively, and the mean value $T_{\mathrm{d}}=19.7 \mathrm{~K}$ for the entire sky (Planck Collaboration et al. 2014a), even though $G_{0}$ in BL is significantly raised above the average interstellar radiation field of the solar neighborhood (=1.7 Habing field; Tielens 2005). For comparison, at $G_{0}=14$ a "typical" $0.1 \mu \mathrm{m}$ silicate (graphite) grain would be heated toward $T_{\mathrm{d}}=19.2(22.5) \mathrm{K}$ (Tielens 2005). At $G_{0}=11$, this would be $T_{\mathrm{d}}=18.6(21.8) \mathrm{K}$. In this respect, Planck Collaboration et al. (2014a) and Planck Collaboration et al. (2014b) argue that observed dust temperatures do not simply trace the intensity of the radiation field but also reflect changes in grain properties, such as the size distribution, grain structure, and material changes. In any case, for both $\mathrm{BL}_{\text {top }}$ and $\mathrm{BL}_{\text {bot }}$, we do not see the necessity of an extra heating source to account for the observed grain temperatures, such as Ly $\alpha$ heating (Heiles et al. 2000).

\subsection{Dust and Gas in the Orion-Eridanus Superbubble}

\subsubsection{Global Observational Characteristics}

The all-sky surveys listed in Section 2 allow us to determine the global observational characteristics of the Orion-Eridanus superbubble, which is of potential use to the study of more distant superbubbles that lack the spatial information of the Orion-Eridanus region. We use apertures that encompass the entire superbubble, the OMCs, and Barnard's Loop, respectively. For the OMCs and Barnard's Loop, these apertures coincide with the outlines depicted in Figure 1(d). While the full extent of the Orion-Eridanus superbubble will be thoroughly discussed in the remainder of this paper, here we already choose the "entire superbubble aperture" to encompass the majority of the $\mathrm{H} \alpha$ emission observed in Figure 1(b), while tracing the outside of $\mathrm{H}$ I filaments seen at $-40 \mathrm{~km} \mathrm{~s}^{-1}>v_{\mathrm{LSR}}$ $>40 \mathrm{~km} \mathrm{~s}^{-1}$ (Figure 1(c)), corresponding to the velocity extent in which the Orion-Eridanus superbubble has previously been identified (Brown et al. 1995). We measure the $\mathrm{H} \alpha$ luminosity and total far-IR to submillimeter luminosity contained within the apertures, which are compared with stellar parameters of Orion OB1.

For the entire superbubble, we proceed as follows. The north and east sides of the superbubble aperture extend close to the Galactic plane and high-latitude clouds. The encompassed IR emission will therefore be contaminated by emission along the line of sight not related to the superbubble. While disentangling the separate contributions to the IR is not straightforward, we attempt to isolate the emission from our region of interest by measuring the average far-IR integrated intensity, $I_{\mathrm{IR}, \text { fil }}$, within the aperture labeled as "IR fil." (Figure 1 (d)), i.e., the dusty shell that encapsulates the limb-brightened Eridanus B and C filaments known to be associated with the superbubble. The intensities are taken from the Planck R1.20 dust model (Planck Collaboration et al. 2014a) that uses IRAS $100 \mu \mathrm{m}$ and Planck 857, 545, and $353 \mathrm{GHz}$ data to determine the total integrated far-IR intensity. Subsequently, we define emission from regions that are much brighter than observed in the limb-brightened filaments, i.e., $I_{\mathrm{IR}} \geqslant 3 I_{\mathrm{IR} \text {,fil }}$, as not being part of the superbubble. This procedure provides a mask tracing the regions of high IR brightness toward the Galactic plane and high-latitude foreground and background clouds well. Note
Table 1

Global Observational Characteristics of the Orion-Eridanus Superbubble

\begin{tabular}{|c|c|c|c|c|c|}
\hline Region & $\begin{array}{c}T_{\mathrm{d}} \\
(\mathrm{K})\end{array}$ & $\begin{array}{c}L_{\mathrm{IR}} \\
\left(10^{5} L_{\odot}\right)\end{array}$ & $\xi_{\star}$ & $\begin{array}{c}L_{\mathrm{Ly} \alpha} \\
\left(10^{5} L_{\odot}\right)\end{array}$ & $\xi_{\text {ion }}$ \\
\hline Superbubble & 19.5 & $7.9(5.7)^{\mathrm{a}}$ & $0.47(0.34)$ & 1.08 & 0.94 \\
\hline $\mathrm{OMC}$ & 17.9 & $2.8(2.9)$ & $0.16(0.17)$ & 0.16 & 0.14 \\
\hline Barnard's Loop & 19.6 & $0.5(0.6)$ & $0.03(0.04)$ & 0.20 & 0.17 \\
\hline
\end{tabular}

Notes. Listed are the dust temperature, $T_{\mathrm{d}}$; the infrared luminosity, $L_{\mathrm{IR}}$; fraction of $L_{\mathrm{IR}}$ to the total luminosity of Orion $\mathrm{OB} 1, \xi_{\star}$; luminosity measured from $\mathrm{H} \alpha$ and converted to $\operatorname{Ly} \alpha, L_{\mathrm{Ly} \alpha}$; and fraction of $\operatorname{Ly} \alpha$ photons to the total amount of

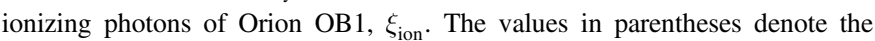
values measured when the masked part of the superbubble is set to zero (see the text). Uncertainties on derived numbers are discussed in the text.

a The total IR luminosity in the superbubble + OMC + Barnard's Loop apertures.

that this procedure filters out the emission from, e.g., the OMCs as well; we ultimately add the contributions of the OMC and Barnard's Loop apertures to get the full IR intensity of the superbubble and the relative contributions of the components (see Table 1). For the $\mathrm{H} \alpha$ map, the background contamination is much less severe and a masking procedure is not necessary. In this respect, we will argue below that all of the observed $\mathrm{H} \alpha$ in Figure 1(b) is caused by photoionization from Orion OB1.

While the masking procedure accounts for the high IR intensities of contaminating clouds toward the Galactic plane, it does not correct for the diffuse low-level background observed over the entire images. Therefore, we apply a global background subtraction to both the far-IR and $\mathrm{H} \alpha$ maps by measuring the background level within a circular region of $0: 4$ radius at $(l, b)=(229.6,-33.7)$ outside of the superbubble aperture that appears to be free of obvious emission features and provides a proper representation of the diffuse background emission averaged over latitude. For both maps, we do not include the $\lambda$ Ori region in our analysis as it appears to be encircled by an ionization-bounded $\mathrm{H}$ iा region (Warren \& Hesser 1977; Wall et al. 1996) and can be regarded as being separated from Orion OB1.

Table 1 lists two different values of $L_{\mathrm{IR}}$. The first value is obtained by filling the masked region with the average IR brightness from the unmasked part of the superbubble aperture. In this way, we get an estimate of the full IR intensity of the entire superbubble if it were unaffected by foreground or background emission along the line of sight. The second value is obtained by setting the masked part to zero, therefore neglecting the masked part of the superbubble. The choice of including or excluding the masked part of the superbubble affects the inferred luminosities from the OMC and Barnard's Loop as well, as these apertures are completely immersed in the masked region of the image, and the chosen intensity of the masked region thus effectively acts as a "global" background for both regions. Below we include the masked region in our interpretations, as we believe that the inclusion of this part of the superbubble is essential to the analysis of the total energetics of the region. However, we refer the reader to Table 1 for the implications of this choice and its impact on the derived parameters. The total far-IR luminosity is $L_{\mathrm{IR}}=4 \pi I_{\text {tot }}$ $S$, where $I_{\text {tot }}$ and $S$ are the total integrated intensity and projected surface area of the emitting region, respectively. For simplicity, we assume that the entire bubble is located at 400 pc. The measured IR luminosity is compared with stellar 
parameters of Orion OB1 through $\xi_{\star}=L_{\mathrm{IR}} / L_{\star}$, where $L_{\star}$ is the total luminosity of the Orion OB region $\left(1.7 \times 10^{6} L_{\odot}\right.$, reevaluated at $d=400 \mathrm{pc}$, and excluding $\lambda$ Ori; Warren $\&$ Hesser 1977; Wall et al. 1996). The parameter $\xi_{\star}$ defines the fraction of stellar radiation that is captured by the dust and reemitted in the IR averaged over all solid angles.

The Ly $\alpha$ photon rate, $N_{\mathrm{Ly} \alpha}$, is calculated by converting the observed $\mathrm{H} \alpha$ photon rate to an $\operatorname{Ly} \alpha$ photon rate using the ratio $\alpha_{\mathrm{B}} / \alpha_{\mathrm{H} \alpha}$, where $\alpha_{\mathrm{H} \alpha}$ is the effective recombination coefficient of $\mathrm{H} \alpha\left(1.31 \times 10^{-13} \mathrm{~cm}^{3} \mathrm{~s}^{-1}\right.$ at $T=6000 \mathrm{~K}$, which is the electron temperature in Barnard's Loop and the Eridanus filaments from Heiles et al. 2000; Madsen et al. 2006; O'Dell et al. 2011; we use this value throughout the superbubble), and $\alpha_{\mathrm{B}}$ is the total recombination coefficient of hydrogen to all levels but the ground state $\left(2.6 \times 10^{-13} \mathrm{~cm}^{-3} \mathrm{~s}^{-1}\right.$; Osterbrock \& Ferland 2006). Case B recombination requires that $N_{\text {Ly } \alpha}$ equals the total amount of recombinations, which is a quantity that can directly be compared to Orion OB1 through $\xi_{\text {ion }}=N_{\mathrm{Ly} \alpha} /$ $N_{\text {ion }}$, where $N_{\text {ion }}$ is the total number of ionizing photons from Orion OB1 $\left(2.7 \times 10^{49}\right.$ photons s ${ }^{-1}$, O'Dell et al. 2011). This ratio measures the fraction of ionizing photons captured by the gas and converted to Ly $\alpha$. In order to directly compare with the IR luminosity $L_{\mathrm{IR}}$, we convert $N_{\mathrm{Ly} \alpha}$ to a luminosity through $L_{\mathrm{Ly} \alpha}=N_{\mathrm{Ly} \alpha} h \nu_{\alpha}$, where $h \nu_{\alpha}$ is the Ly $\alpha$ photon energy, and define $L_{\text {ion }}=N_{\text {ion }} h v_{\alpha}$, such that $\xi_{\text {ion }}=N_{\text {Ly } \alpha} / N_{\text {ion }}=L_{\text {Ly } \alpha} / L_{\text {ion }}$.

Absolute uncertainties on the $\mathrm{H} \alpha$ intensity map are less than 10\% (Finkbeiner 2003), while Planck (2011) reports an uncertainty of $\sim 2 \%$ for the Planck $353 \mathrm{Ghz}$ channel measurements. However, total errors for the values reported in Table 1 are also dependent on the determination of the background level, as well as the adopted values for Orion OB1 $\left(L_{\star}\right.$ and $\left.L_{\text {ion }}\right)$. We estimate a $10 \%$ uncertainty on the values of $L_{\mathrm{IR}}, \xi_{\star}, L_{\mathrm{Ly} \alpha}$, and $\xi_{\text {ion }}$, denoted in Table 1 . The error on the dust temperature $T_{\mathrm{d}}$ measured from the Planck R1.20 dust model is of order of 3\% within bright regions of the sky (Abergel 2013) such as the OMC and Barnard's Loop apertures, whereas the error is somewhat higher $(8 \%)$ in regions that contain diffuse emission such as the Orion-Eridanus superbubble aperture.

The calculated luminosities, $L_{\mathrm{IR}}$ and $L_{\mathrm{Ly} \alpha}$, are denoted in Table 1. Table 1 shows that half of the total amount of stellar radiation of Orion OB1 is trapped in the superbubble and reradiated in the IR, $\xi_{\star} \sim 0.5$. The molecular clouds account for about $35 \%$ of the total IR emission of the superbubble; the contribution from Barnard's Loop is negligible. In contrast, $\xi_{\text {ion }}$ of the OMC (0.14) and Barnard's Loop (0.17) are roughly similar, which is because the Orion Nebula and IC 434 emission nebula are currently breaking out of the molecular clouds (Section 3.3) and are contained within the OMC aperture. Eventually, all ionizing photons are absorbed within the superbubble aperture $\left(\xi_{\text {ion }} \sim 1\right)$. This implies that, on average, Orion OB1 can provide the necessary ionizing power to illuminate the $\mathrm{H} \alpha$ structures detected in Figure 1(c), including the $\mathrm{H} \alpha$ filaments that run along the outer edge of the superbubble aperture. Nonetheless, it is possible that individual small-scale structures may still be too bright given their size and distance from Orion OB1 (Pon et al. 2014a). Note that some of these filaments lie outside of what has previously been thought to be the edges of the Orion-Eridanus superbubble (Reynolds \& Ogden 1979; Heiles et al. 1999; Bally 2008; Pon et al. 2014b). The dust temperatures measured inside the superbubble aperture and Barnard's Loop are similar to that observed for the entire diffuse sky by Planck (see discussion in Section 3.1.2). Inside the OMC, this value is somewhat lower, which is what is expected for dense regions (Planck Collaboration et al. 2014a).

\subsubsection{Tracing the Superbubble Structure through Dust and Gas}

To investigate the gas and dust content in specific regions throughout the Orion-Eridanus region, we use apertures depicted in Figure 1 that are defined such that the majority of $\mathrm{H} \alpha$ and far-IR emission from the regions is enclosed (for example, the stratified emission from the limb-brightened emission of the superbubble wall; Figure 2(d)), while a low background level is ensured through comparison with the Planck and LAB maps (the $\mathrm{H} \alpha$ emission does not show a high background in general). As the diffuse background emission varies significantly over the entire Orion-Eridanus region, here we use local background values to properly measure the luminosities contained in the apertures scattered over the Orion-Eridanus region. For the apertures at $b>-30^{\circ}$, we define a background at $(l, b)=(233.2,-28.6)$ within a circle of 0.4 radius. For the apertures at $b<-30^{\circ}$, we define a background at $(l, b)=(221.9,-49.5)$. Region 5 is an exception to this rule, as the previously defined background levels were not representative of the local value because of its location among high-latitute clouds. For region $5,(l, b)=(176.3$, $-15.0)$ was chosen.

Ly $\alpha$ photons will be resonantly scattered many times in the $\mathrm{H}$ II region because of its large line cross section, but they eventually be absorbed by dust, contributing to the heating of the dust. The ratio of heating rate by stellar photons, $\Gamma_{U V}$, to Ly $\alpha$ photons, $\Gamma_{\mathrm{Ly} \alpha}$, can be written as (Tielens 2005)

$$
\frac{\Gamma_{\mathrm{UV}}}{\Gamma_{\mathrm{Ly} \alpha}}=\frac{\pi a^{2} n_{\mathrm{d}} \bar{Q}_{\mathrm{abs}} L_{\star}}{4 \pi r^{2} n^{2} \alpha_{\mathrm{B}} h \nu_{\alpha}} .
$$

Here $\pi a^{2}$ and $n_{\mathrm{d}}$ are the geometrical cross section and number density of the grains, respectively, $\bar{Q}_{\mathrm{abs}}$ is the average radiation absorption efficiency of the dust, $L_{\star}$ is the total luminosity of Orion OB1, $r$ is the distance to the source, and $n$ is the hydrogen number density of the gas. Assuming equilibrium between photoionization and recombination, for a shell surrounding an empty cavity we have $f_{\text {ion }} N_{\text {ion }}=4 \pi r^{2} n^{2} \alpha_{\mathrm{B}}$ $\Delta r$, where $f_{\text {ion }}$ is the fraction of incident ionizing photons available that are absorbed by the gas locally. Then, Equation (1) reduces to

$$
\frac{\Gamma_{\mathrm{UV}}}{\Gamma_{\mathrm{Ly} \alpha}}=\frac{\pi a^{2} n_{\mathrm{d}} \bar{Q}_{\mathrm{abs}} \Delta r}{f_{\text {ion }}}\left(\frac{L_{\star}}{N_{\mathrm{ion}} h \nu_{\alpha}}\right)=\frac{\tau_{\mathrm{d}}}{f_{\text {ion }}}\left(\frac{L_{\star}}{N_{\text {ion }} h \nu_{\alpha}}\right),
$$

where $\tau_{\mathrm{d}}$ is the (radial) absorption optical depth of the shell. With $L_{\star}=1.7 \times 10^{6} L_{\odot}$ and $N_{\text {ion }}=2.7 \times 10^{49}$ photons s $^{-1}$ (Section 3.2.1), we have $\left(L_{\star} / N_{\text {ion }} h \nu_{\alpha}\right) \approx 15$ for Orion OB1, and therefore the ratio $\Gamma_{\mathrm{UV}} / \Gamma_{\mathrm{Ly} \alpha}$ equals unity for $\tau_{\mathrm{d}} / f_{\text {ion }} \approx 0.07$.

We can make a priori estimates of $f_{\text {ion }}$ for the regions defined in Figure 1. For Barnard's Loop, we have measured an average $\xi_{\text {ion }}=0.17$ over the entire structure (Table 1 ). A factor of 0.50 would be expected for a half-sphere geometry if Barnard's Loop were optically thick to ionizing photons. Possibly, a fraction of the ionizing photons from Orion OB1 is trapped within the OMCs before reaching Barnard's Loop, which would lead to $\xi_{\text {ion, BL }}=0.50-\xi_{\text {ion, OMC }}=0.36$ (Table 1). Still, these numbers reveal that $50 \%-66 \%$ of the ionizing photons 

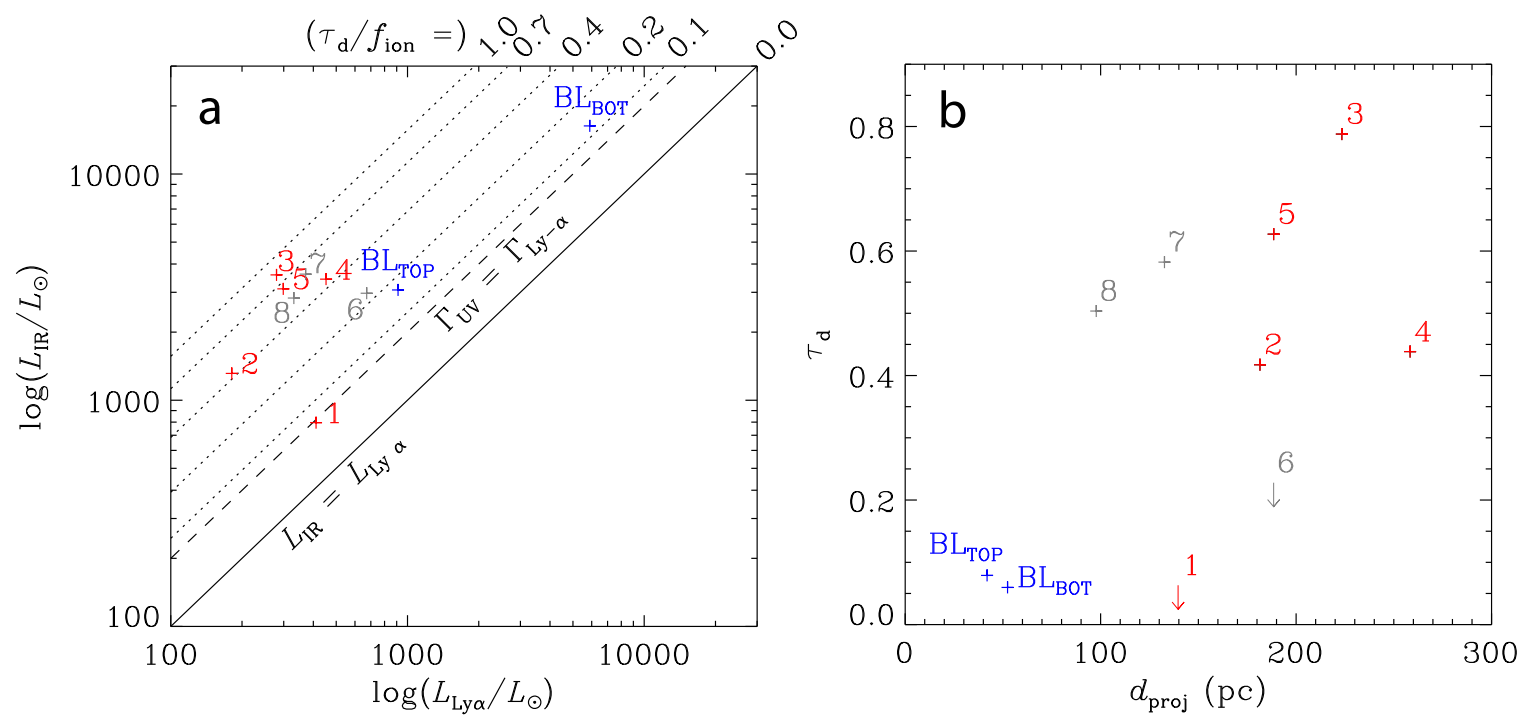

Figure 3. Apertures tracing the structure of the Orion-Eridanus superbubble. (a) Infrared vs. Ly $\alpha$ luminosities measured in the apertures shown in Figure 1. The solid line shows where the infrared and Ly $\alpha$ luminosities are equal. The lower dashed line shows where the infrared and Ly $\alpha$ heating rates are equal, corresponding to $\tau_{\mathrm{d}} /$ $f_{\text {ion }}=0.07$ (see the text), where $\tau_{\mathrm{d}}$ is the dust optical depth at UV wavelengths and $f_{\text {ion }}$ is the fraction of ionizing photons available absorbed by the gas. The dotted lines are similar, but for increasing $\tau_{\mathrm{d}}$. Overplotted are the luminosities measured in the apertures drawn in Figure 1, color-coded as follows: regions 1-5 (red) are positioned along the projected outer shell of the superbubble aperture (Figure 1(c)); Barnard's Loop $\left(\mathrm{BL}_{\text {top }}\right.$ and $\mathrm{BL}_{\text {bot }}$ ) (blue); regions 6, 7, and 8 are apertures located on the Eridanus A filament, the G203-37 molecular cloud, and the G204-31 molecular cloud, respectively (gray; see the text). (b) Radial dust optical depth at UV wavelengths vs. the projected distance toward the $\mathrm{H} \alpha$ flux-weighted center of the superbubble.

pass through the half sphere encompassing Barnard's Loop. Hence, we estimate that $f_{\text {ion }}$ is in the range $\sim 0.33-0.50$ for the Barnard's Loop apertures $\mathrm{BL}_{\text {bot }}$ and $\mathrm{BL}_{\text {top. }}$. The fact that Barnard's Loop is optically thin for the ionizing flux of Orion OB1 can also be inferred from the morphological appearance of the cometary clouds L1617 and L1622, which are located behind Barnard's Loop as measured from the Orion OB association and have sharp I-fronts on their sides facing the Belt stars (Bally et al. 2009). We note that the internal structure of Barnard's Loop may be more complex than that of a homogeneously distributed half-sphere assumed here. A patchy structure containing optically thick clumps $\left(f_{\text {ion }} \sim 1\right)$ and holes through which the photons would leak unhindered $\left(f_{\text {ion }} \sim 0\right)$ could, in principle, lead, on average, to an $f_{\text {ion }}$ of order $0.33-0.50$. However, Barnard's Loop, similar to region 1 and region 6 , is not associated with $\mathrm{H} \mathrm{I}$. The remaining regions are associated with neutral hydrogen, where $f_{\text {ion }}$ will be of order $\sim 1$ because of the small mean free path of ionizing photons through $\mathrm{H}_{\mathrm{I}}$ (Tielens 2005).

We measure both $L_{\mathrm{IR}}$ and $L_{\mathrm{Ly} \alpha}$ within the apertures shown in Figure 1 and compare these measurements with luminosities from Orion OB1. The structures contained in each defined aperture will subtend a solid angle $\Omega$ as measured from Orion OB1. Now, any ionizing photon that is absorbed by hydrogen and converted to Ly $\alpha$ will eventually contribute to the heating of the dust, and therefore the total IR luminosity that emanates from each region can be written as

$$
L_{\mathrm{IR}}=L_{\mathrm{UV}}+L_{\mathrm{Ly} \alpha}=\tau_{\mathrm{d}} L_{\star} \Omega_{\mathrm{UV}}+f_{\text {ion }} N_{\mathrm{ion}} h \nu_{\alpha} \Omega_{\mathrm{Ly} \alpha},
$$

where $L_{\mathrm{UV}}$ is the dust luminosity provided by direct absorption of stellar photons. In the classical picture of an expanding superbubble, a dense, neutral shell will wrap around the $10^{4} \mathrm{~K}$ region created by the reverse shock traced by bright $\mathrm{H} \alpha$ emission (Weaver et al. 1977). Therefore, we can assume that the IR and $\mathrm{H} \alpha$ trace structures that measure the same solid angle with respect to Orion $\mathrm{OB} 1, \Omega_{\mathrm{UV}}=\Omega_{\mathrm{Ly} \alpha}$, and we can write

$$
\frac{\left(L_{\mathrm{IR}} / L_{\star}\right)}{\left(L_{\mathrm{Ly} \alpha} / L_{\text {ion }}\right)}=\frac{\tau_{\mathrm{d}}}{f_{\text {ion }}}+\left(\frac{N_{\text {ion }} h \nu_{\alpha}}{L_{\star}}\right),
$$

which describes the ratio between the amount of energy that is captured by the dust and reemitted in the IR and the amount of energy that is captured by the gas and converted to Ly $\alpha$.

Our results are shown in Figure 3. All studied regions show $L_{\mathrm{IR}}>L_{\mathrm{Ly} \alpha}($ Figure $3(\mathrm{a}))$, which reveals that a (substantial) amount of stellar radiation is directly absorbed by the dust. Here we note that $L_{\mathrm{IR}}=2 L_{\mathrm{Ly} \alpha}$ when $\Gamma_{\mathrm{UV}}=\Gamma_{\mathrm{Ly} \alpha}$ (see Equation (3)). An interesting trend can be seen from Figure 3 (a). Regions 2-5, positioned on filamentary structures seen in gas and dust along the outside of the superbubble aperture depicted in Figure 1, reveal significantly higher fractions of $\tau_{\mathrm{d}} /$ $f_{\text {ion }}$ compared to the regions located in Barnard's Loop $\left(\mathrm{BL}_{\mathrm{bot}}\right.$, $\left.\mathrm{BL}_{\text {top }}\right)$. This implies that stellar photons, provided by Orion OB1 (Section 3.2.1), are more efficiently absorbed in regions 2-5 compared to those in Barnard's Loop. In other words, the dust optical depth for stellar photons associated with Barnard's Loop is very small. In Figure 3(b), we rewrite Equation (4) for $\tau_{\mathrm{d}} / f_{\text {ion }}$ and, together with our estimations for $f_{\text {ion }}$ as described above, plot the derived dust optical depth in the regions as a function of projected distance $d_{\text {proj }}$ from $(l, b)=(206.5$, $-18.0)$, the flux-weighted center of the bubble as measured from the $\mathrm{H} \alpha$ emission (see Figure 1(b); Reynolds \& Ogden 1979). We exclude $\lambda$ Ori as it seems to be encircled by an ionization-bounded $\mathrm{H}$ II region. Still, in Section 3.3.3 we show that the ionized gas in the $\lambda$ Ori region exhibits the characteristics of a champagne flow (Tenorio-Tagle 1979), in which case the $\mathrm{H}$ II region will be density bounded on the side of the outward champagne flow, and radiation will likely be able to escape the region. However, the circular morphology of 

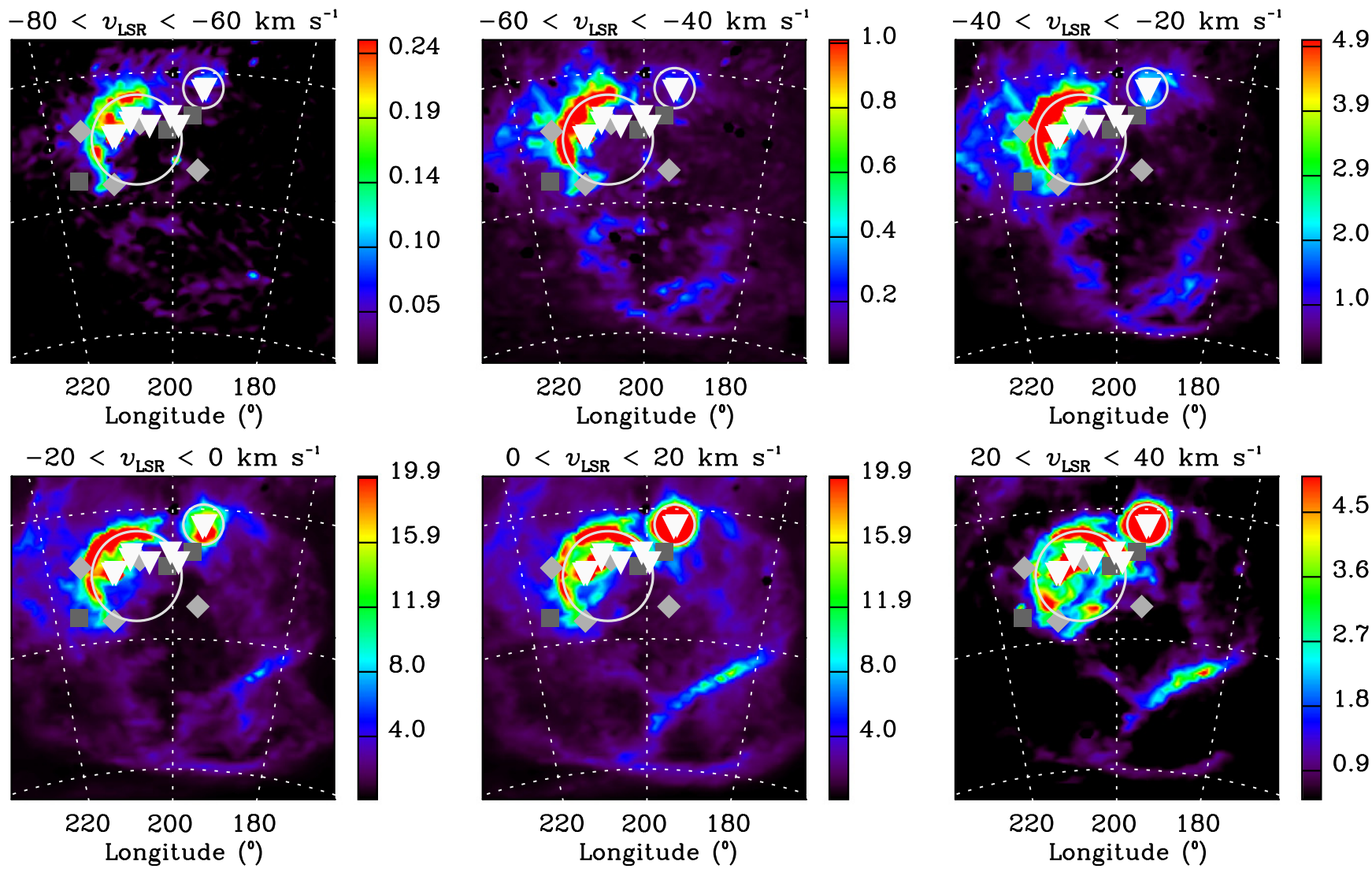

$220 \quad 200 \quad 180$ Longitude $\left(^{\circ}\right)$ Longitude $\left({ }^{\circ}\right)$
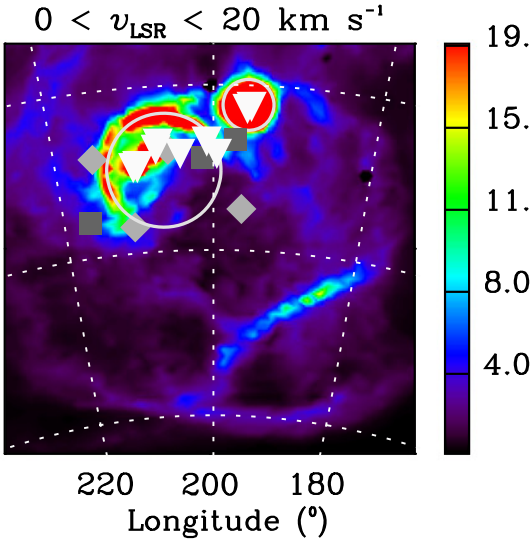

$60<v_{\mathrm{LSR}}<80 \mathrm{~km} \mathrm{~s}^{-1}$

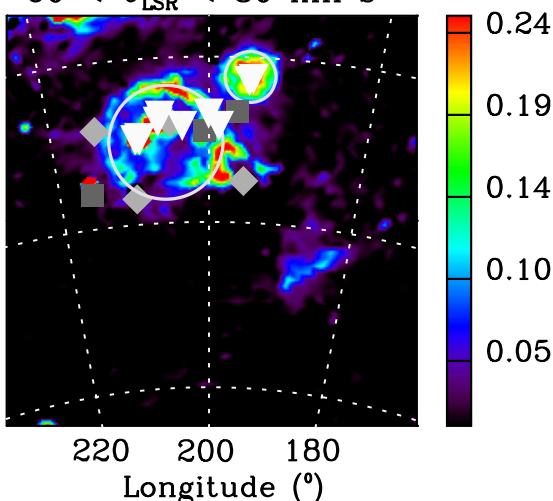

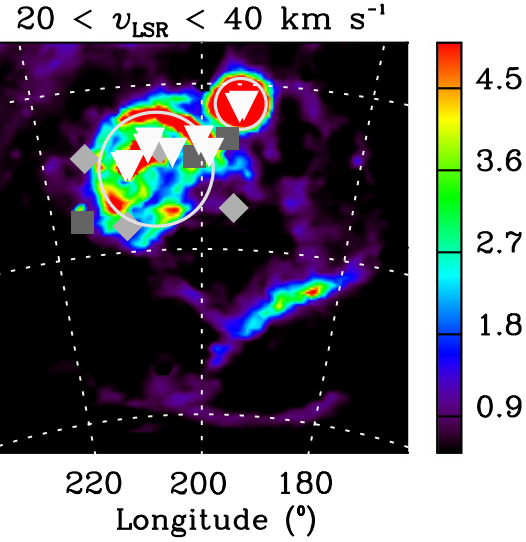

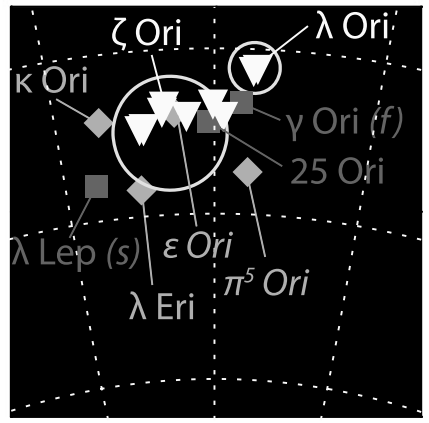

$220 \quad 200 \quad 180$ Longitude $\left({ }^{\circ}\right)$

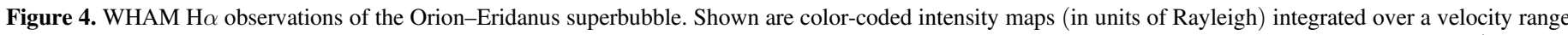

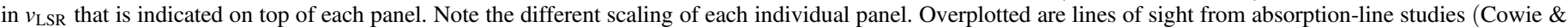

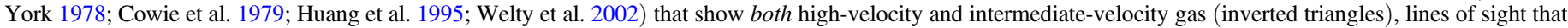

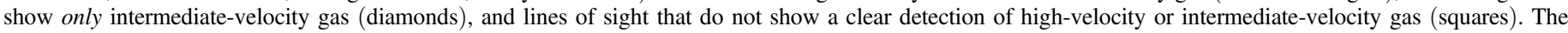

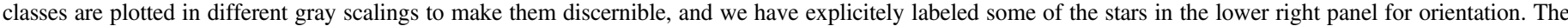

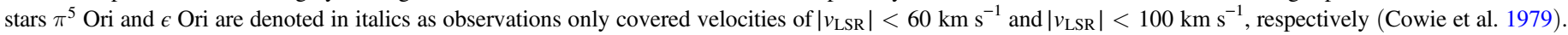

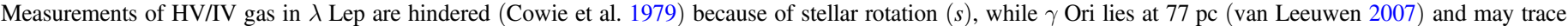
foreground gas $(f)$.

the $\lambda$ Ori bubble detected in $\mathrm{H} \alpha$ (Figure 4) reveals that the champagne phase, if present, may not have fully developed (Section 3.3.3), and therefore we will assume that the $\lambda$ Ori $\mathrm{H}$ II region is still ionization bounded.

For the BL apertures, we plot the results for $\tau_{\mathrm{d}}$ versus $d_{\text {proj }}$ assuming $f_{\text {ion }}=0.5$ (Section 3.2.2). For regions 1 and 6, the absence of a clear neutral $\mathrm{H}_{\mathrm{I}}$ filament prohibited us from predicting $f_{\text {ion }}$, and only an upper limit to $\tau_{\mathrm{d}}$ is given. The results plotted in Figure 3(b), albeit suffering from low-number statistics, indicate a trend where $\tau_{\mathrm{d}}$ rises with increasing $d_{\text {proj. }}$.
From Figure 3, it becomes apparent that dust associated with Barnard's loop is not able to capture all of the radiation emitted by the Orion OB1 association. In this context, the bright $\mathrm{H} \alpha$ filament to the southeast that surrounds Barnard's Loop, from region 1 up to the Galactic plane (Figure 1), is lit up by ionizing photons breaking through Barnard's Loop. A straight line from the flux-weighted center of the superbubble (Figure 1), through Barnard's Loop to the outer filament in region 1 , reveals a flux ratio of $F_{\mathrm{BL}} / F_{\text {reg. } 1} \sim 5$, where $F_{\mathrm{BL}}$ and $F_{\text {reg. } 1}$ are the measured peak $\mathrm{H} \alpha$ fluxes in Barnard's Loop and 
the outer filament in region 1 , respectively. At a distance ratio of $d_{\mathrm{BL}} / d_{\text {reg.1 }} \sim 2.5$, this is consistent with both the structures absorbing a similar amount of ionizing photons, confirming $f_{\text {ion }}$ $\sim 0.5$ for the Barnard's Loop apertures, as was assumed in our derivations of $\tau_{\mathrm{d}}$ above.

As a sanity check to our methodology, we also plot in Figure 3 the results for region 6 (corresponding to the southern end of the Eri A filament), which reveals low $\tau_{\mathrm{d}}$ compared to the other Eridanus filaments. However, the association of the Eridanus A filament with the superbubble is still controversial and is discussed thoroughly in Pon et al. (2014a). For completeness, we show the results for apertures located on the G203-37 and GS204-31 clouds (regions 7 and 8), which are thought to be inside and at the near side of the OrionEridanus superbubble (Snowden et al. 1995). The clouds are highly opaque to $0.25 \mathrm{keV}$ radiation with intervening hydrogen column densities of $\sim 8 \times 10^{20} \mathrm{~cm}^{2}$ (Snowden et al. 1995), translating to an optical depth at UV wavelength of $\tau_{\mathrm{UV}} \sim 0.8$ using the relations given in Section 3.1.1, in rough agreement with our measurements shown in Figure 3.

With $f_{\text {ion }}=0.5$ for the Barnard's Loop apertures, the dust optical depth as measured from the IR $\left(\tau_{\mathrm{d}}=0.06\right.$ and $\tau_{\mathrm{d}}=0.08$ for $\mathrm{BL}_{\text {top }}$ and $\mathrm{BL}_{\text {bot }}$, respectively; Figures $3(\mathrm{a})$ and (b)) is in agreement with a homogeneous shell containing dust similar to that seen in the diffuse ISM $\left(\tau_{\mathrm{UV}}=0.07\right.$; Section 3.1$)$. Finally, we note that while Ly $\alpha$ contributes about $25 \%-50 \%$ of the total energy absorbed by dust in Barnard's Loop (Figure 3(a)), this will have little effect on the dust temperature (Section 3.1.2) as $T_{\mathrm{d}}$ only weakly depends on the heating rate. In particular, $T_{\mathrm{d}} \propto$ $\left(\Gamma_{\mathrm{UV}}+\Gamma_{\mathrm{Ly} \alpha}\right)^{1 / 6}$ for silicate grains (Tielens 2005).

\subsection{Expanding Shells within the Orion-Eridanus Region}

\subsubsection{High- and Intermediate-velocity Gas}

The presence of $\mathrm{HV}$ absorption features of ionized gas between $-120 \mathrm{~km} \mathrm{~s}^{-1} \lesssim v_{\text {LSR }} \lesssim-80 \mathrm{~km} \mathrm{~s}^{-1}$ was detected in multiple lines of sight toward Orion (Cowie et al. 1979; Welty et al. 2002). In addition, IV features were detected between $-80 \mathrm{~km} \mathrm{~s}^{-1} \lesssim v_{\mathrm{LSR}} \lesssim-20 \mathrm{~km} \mathrm{~s}^{-1}$ (Cowie \& York 1978; Cowie et al. 1979; Huang et al. 1995; Welty et al. 2002). Both components show similar depletions, ion ratios, and physical conditions, albeit the IV gas contains column densities that exceed that of the HV gas by a factor of 3 (e.g., Welty et al. 2002). The HV gas, dubbed by Cowie et al. (1979) as "Orion's Cloak," has an angular diameter of at least $15^{\circ}$ and is ascribed to a recent $\mathrm{SN}$ that, from its dynamical expansion scale, should have occurred some $3 \times 10^{5} \mathrm{yr}$ ago.

Figure 4 shows $\mathrm{H} \alpha$ velocity maps from the WHAM $\mathrm{H} \alpha$ spectral survey (Haffner et al. 2003) of the Orion-Eridanus superbubble, covering the velocity space between $-80 \mathrm{~km} \mathrm{~s}^{-1}$ $\lesssim v_{\mathrm{LSR}} \lesssim+80 \mathrm{~km} \mathrm{~s}^{-1}$. Overplotted are sightlines from absorption-line studies (Cowie \& York 1978; Cowie et al. 1979; Huang et al. 1995; Welty et al. 2002). The HV gas and IV gas trace structures around Barnard's Loop and the $\lambda$ Ori ring that extend outside of the traditional limits of the Orion-Eridanus superbubble (Reynolds \& Ogden 1979; Heiles et al. 1999; Bally 2008; Pon et al. 2014b), as demonstrated by the sightlines of $\kappa$ Ori, $\lambda$ Ori, and $\lambda$ Eri. While it must be recognized that the distribution of the gases is somewhat patchy (Cowie et al. 1979), it is clear that the IV gas and HV gas trace two distinct components. Moreover, the HV gas seems to be confined to a more limited area compared to the IV gas, as reflected by the sightlines that show only the IV gas.

We note upfront that electron temperatures within Barnard's Loop and $\lambda$ Ori are determined at $\sim 6000-7500 \mathrm{~K}$ (Reich 1978; Heiles et al. 2000; Madsen et al. 2006; O’Dell et al. 2011), which excludes that the $\mathrm{HV}$ gas originates from $\mathrm{HV}$ wings of a thermal Gaussian line profile (the thermal broadening can be estimated with $\sqrt{2 k T / m_{\mathrm{H}}} \sim 10 \mathrm{~km} \mathrm{~s}^{-1}$, where $k$ is Boltzmann's constant and $m_{\mathrm{H}}$ is the mass of a hydrogen atom). The velocity information of the Eridanus filaments is discussed in Pon et al. (2014a). Of particular interest here are the encircled regions toward the east of the superbubble, covering the Barnard's Loop region and the $\lambda$ Ori region, as well as smaller-scale bubbles.

\subsubsection{Barnard's Loop Bubble}

The intensity peak of Barnard's Loop lies between $-20 \mathrm{~km} \mathrm{~s}^{-1} \lesssim v_{\mathrm{LSR}} \lesssim+20 \mathrm{~km} \mathrm{~s}^{-1}$, but the structure is visible throughout the entire velocity space covered by the WHAM survey. Here we reveal the presence of an ionized filament, visible only at positive velocities $v_{\mathrm{LSR}}>+20 \mathrm{~km} \mathrm{~s}^{-1}$. This filament appears to form a complete bubble structure together with the bright crescent of Barnard's Loop, centered on ( $l$, $b)=(193,-20)$ with radius $14^{\circ}$. The lines of sight covered by the absorption survey of Cowie et al. (1979) indicate that the $\mathrm{HV}$ gas originates from the encircled $\mathrm{H} \alpha$ bubble structure, or the "Barnard's Loop Bubble" (Figure 4), suggesting a connection between both components. Cowie et al. (1979) estimated the size of Orion's Cloak at a minimum of $15^{\circ}$ that matches the size of the Barnard's Loop bubble. Thus, the Barnard's Loop bubble may well be part of the supernova remnant (SNR) associated with Orion's Cloak.

The most pronounced radial velocity effects are expected to originate from the center of an expanding bubble. For the HV gas, these velocities lie outside of the velocity range covered by the WHAM survey $\left(-80 \mathrm{~km} \mathrm{~s}^{-1} \lesssim v_{\text {LSR }} \lesssim 80 \mathrm{~km} \mathrm{~s}^{-1}\right.$; Haffner et al. 2003), rendering WHAM unable to pick up the HV gas detected by Copernicus (Cowie et al. 1979) and Hubble Space Telescope (Welty et al. 2002), although it is questionable whether WHAM would be able to pick up the low surface brightness of the HV gas expected in $\mathrm{H} \alpha\left(\sim 3 \times 10^{-3}\right.$ Rayleigh; Cowie et al. 1979). The OMCs are roughly centered on $v_{\mathrm{LSR}}$ $=10 \mathrm{~km} \mathrm{~s}^{-1}$ in velocity space (Wilson et al. 2005); if we assume a systemic velocity toward the Orion region of $v_{\mathrm{LSR}}$ $=10 \mathrm{~km} \mathrm{~s}^{-1}$ and connect the HV gas with the expansion of the Barnard's Loop bubble, we estimate its expansion velocity at $v_{\text {exp }}=100 \mathrm{~km} \mathrm{~s}^{-1}$. However, it is not clear how much of the mass within the Barnard's Loop bubble (Section 4.3.1) is associated with this expansion velocity. The coverage of sightlines of the Orion-Eridanus region is limited, and the HV gas could in principle trace small, HV components related to the Barnard's Loop bubble, rather than tracing the bulk expansion of the bubble itself. Here we implicitly assume that the bulk of the gas in the expanding shell is expanding at the adopted expansion velocity. The preshock densities for the HV gas toward $\zeta$ Ori $\left(n_{0}=3 \times 10^{-3} \mathrm{~cm}^{-3}\right.$; Welty et al. 2002) then imply that the bubble expands into either the hot intercloud medium $\left(T \sim 10^{6} \mathrm{~K}, n \sim 3 \times 10^{-3} \mathrm{~cm}^{-3}\right)$ that pervades the Galactic halo or a preexisting cavity, such as the superbubble interior. We defer a thorough discussion of the Barnard's Loop bubble and its connection to the HV gas to Section 4. 


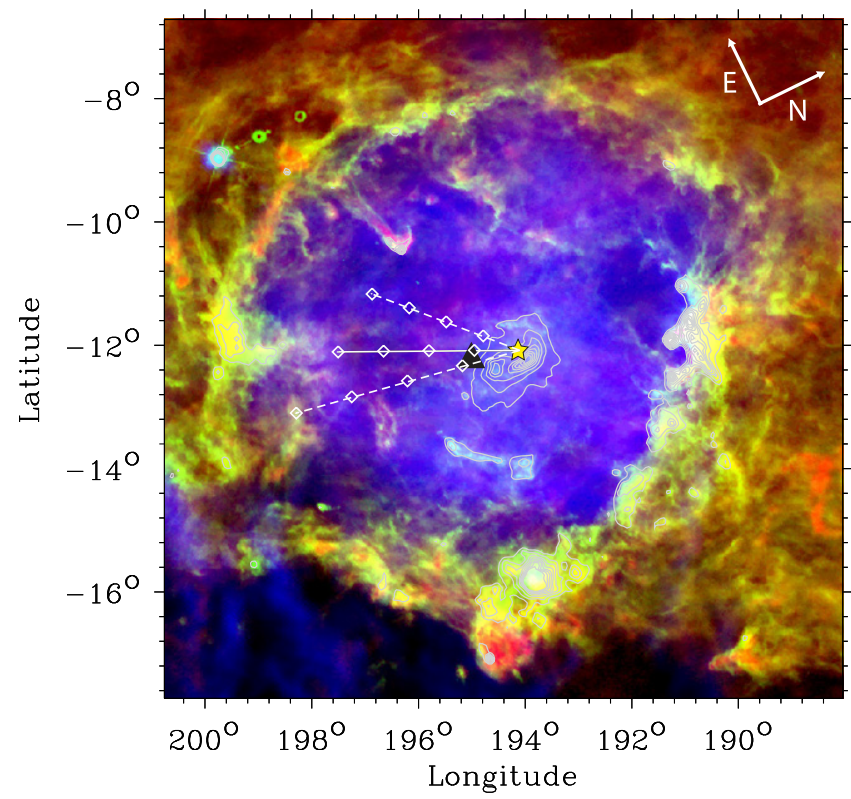

Figure 5. Three-color image of the $\lambda$ Ori bubble. The color codes are the same as in Figure 1a. Overlayed are contours of IRAS $60 \mu \mathrm{m}$, with intensity levels: $100 \%$ (maximum intensity)-90\%-80\%-70\%-60\%-50\%-40\%-30\%. The solid line marks the past trajectory of the star $\lambda$ Ori during 4 Myr given its current space motion. Open diamonds mark intervals of $1 \mathrm{Myr}$. The dashed lines are the extremes of possible space motion considering the errors in proper motion and distance to the star. The black triangle marks the center of expansion of the $\lambda$ Ori bubble (Lang \& Masheder 1998).

\subsection{3. $\lambda$ Ori Bubble}

The circular-symmetric $\mathrm{H}_{\mathrm{II}}$ region surrounding $\lambda$ Ori has been known for a long time. Through a study of the star formation history of the $\lambda$ Ori region, Dolan \& Mathieu (1999) hypothesized that about $1 \mathrm{Myr}$ ago, an SN disrupted the parent molecular cloud and created the giant $\mathrm{H}$ II region seen to date, which is maintained by the remaining population of massive stars in the region. The expansion velocity of the $\mathrm{H}$ II region is traced by observations of the dense molecular shell surrounding the ionized gas and is estimated at $v_{\exp }=16.5 \mathrm{~km} \mathrm{~s}^{-1}$ expanding from the point $(l, b)=(195.8,-12.1)$ centered at $v_{\mathrm{LSR}}$ $=3.8 \mathrm{~km} \mathrm{~s}^{-1}$ in velocity space (Lang \& Masheder 1998).

The main ionizing star $\lambda$ Ori lies right at the (projected) heart of the bubble, which seems inconsistent with its proper motion $\left(\mu_{\alpha} \cos \delta=-0.34 \pm 0.60\right.$ mas and $\mu_{\delta}=-2.94 \pm$ 0.33 mas; van Leeuwen 2007) with respect to the dense, molecular shell (Maddalena \& Morris 1987; Lang \& Masheder 1998). We calculate the LSR space velocity of $\lambda$ Ori following the method described in Cox et al. (2012), using its proper motion (van Leeuwen 2007) and radial velocity $\left(v_{\text {rad }}\right.$ $=30.1 \mathrm{~km} \mathrm{~s}^{-1}$; Gontcharov 2006), while correcting for the solar motion using parameters from Coşkunoğlu et al. (2011). We adopt a distance of $450 \mathrm{pc}$ to the star (Dolan \& Mathieu 2001). The calculated space motion of $\lambda$ Ori is $v_{\text {rad }}$ $=18.2 \mathrm{~km} \mathrm{~s}^{-1}$ with a position angle of $330^{\circ}$ (from north to east) inclined by $55^{\circ}$ in the plane of the sky. The trajectory is overplotted in Figure 5 and reveals that it is unlikely that its current motion has been imparted at birth, since $\lambda$ Ori would have then originated at the boundary between the molecular and ionized gas (the expected age of $\lambda$ Ori is $~ 3-5 \mathrm{Myr}$; see Hernández et al. 2010, and references therein). However, its motion and distance from the center of expansion (Figure 5) are consistent with the SN hypothesis that would have launched $\lambda$ Ori on its current trajectory about 1 Myr ago.

We recognize the arc-shaped mid-infrared emission around $\lambda$ Ori, which is the telltale signature of a dust wave, created by the interaction of dust entrained in a champagne flow with radiation pressure from the star, which is a common phenomenon inside interstellar bubbles (Ochsendorf et al. 2014a, 2014b). Indeed, the radio continuum maps presented by Reich (1978) reveal an emission gradient within the ionized gas that is expected for a champagne flow (TenorioTagle 1979). The emission gradient and the location of the dust wave reveal that the interior of the $\lambda$ Ori bubble is venting into the surrounding medium toward the southeast. However, the overall distribution of the ionized gas is still roughly spherical (Figure 4); an extended emission region that would accompany a developed $\mathrm{H}$ II region champagne flow is not detected. This may indicate that the champagne phase has just commenced and the bubble is on the verge of breaking out. The implications of these observations shall be addressed in Section 4.3.

The WHAM observations in Figure 4 reveal that the $\lambda$ Ori $\mathrm{H}$ II region lights up at positive $v_{\mathrm{LSR}}$ (Figure 4), extending longward of $v_{\mathrm{LSR}}>80 \mathrm{~km} \mathrm{~s}^{-1}$. In addition, elevated emission surrounding the $\lambda$ Ori region can be seen at the other velocity extreme of WHAM, i.e., $-80 \mathrm{~km} \mathrm{~s}^{-1} \lesssim v_{\mathrm{LSR}} \lesssim-60 \mathrm{~km} \mathrm{~s}^{-1}$, possibly tracing the same component from Cowie et al. (1979), who detected faint $\mathrm{HV}$ gas features toward $\lambda$ Ori. The FWHM of the $\mathrm{H} \alpha$ line in the $\lambda$ Ori region is $\sim 80 \mathrm{~km} \mathrm{~s}^{-1}$, much broader than the thermal line width $\left(\sim 10 \mathrm{~km} \mathrm{~s}^{-1}\right.$; Section 3.3.1). Thus, both the $\mathrm{HV}$ gas and $\mathrm{H} \alpha$ suggest higher velocities than the observed expansion velocity of the molecular shell ( $v_{\exp }$ $=16.5 \mathrm{~km} \mathrm{~s}^{-1}$; Lang \& Masheder 1998). The discrepancy between velocities observed for the dense molecular ring and the ionized gas could indicate a fast blister flow (see above), which can lead to large line-of-sight variations, or a recent explosive event that has accelerated the gas in the bubble interior but has not yet coupled to the dense molecular ring.

\subsubsection{GS206-17+13 and the Orion Nebula}

Besides the obvious large-scale shells that are associated with Barnard's Loop and $\lambda$ Ori, we note that the Orion complex harbors shell structures on all detectable scales. Below we discuss the degree-sized GS206-17+13 shell (Ehlerová \& Palouš 2005) and the shell surrounding the Orion Nebula (O'Dell 2001; Güdel et al. 2008), but on even smaller scales there have been detections of many regions within the Orion A cloud that appear to have shell-like structures, presumably driven by $\mathrm{H}$ iI regions from massive young stellar objects (Bally et al. 1987; Heyer et al. 1992). Both GS206-17+13 and the Orion Nebula emit at positive $v_{\text {LSR }}$ (Figure 4) with large line widths (FWHM $\sim 70 \mathrm{~km} \mathrm{~s}^{-1}$ ) that can both be related to ionized (blister) flows as described below.

Ochsendorf \& Tielens (2015) discussed the GS206-17+13 shell, which is directly adjacent to the OMC B, has dimensions of $2^{\circ} \times 4^{\circ}(14 \times 28 \mathrm{pc})$, and is approximately centered on the $\sigma$ Ori star cluster. The formation of the shell is likely caused by the stellar winds of the Orion OB1b subgroup that formed the protrusion out of the OMC B. The bright $\mathrm{H}_{\mathrm{II}}$ emission that is projected inside part of the shell (the IC 434 emission nebula) is not related to the formation or history of GS206-17+13, as this gas originates from the champagne flow driven by the ionizing flux of the $\sigma$ Ori cluster that has entered the 
preexisting bubble cavity and is now approaching OMC B (Ochsendorf \& Tielens 2015). Ionization of the cloud edge sets up a pressure discontinuity between the cloud and intercloud medium and drives a shock front into the low-density bubble, while a rarefaction wave travels into the OMC B cloud. In panel $(2 \mathrm{a})$ of Figure 2, the shock front is visible at $r_{\mathrm{s}}$, whereas the rarefaction wave is located at $r_{\mathrm{rw}}$. Between the shock fronts, a champagne flow of ionized gas is set up (TenorioTagle 1979), flowing from $r_{\mathrm{rw}}$ toward $r_{\mathrm{s}}$.

The density structure of the champagne flow of the IC 434 region is well characterized by an exponential density gradient (Ochsendorf \& Tielens 2015), reaching from $n_{\mathrm{H}}=35 \mathrm{~cm}^{-3}$ at the IF on the OMC B cloud surface to $3.5 \mathrm{~cm}^{-1}$ at the location of $\sigma$ Ori $\left(r_{\star}\right.$; Figure 2), where the flow reaches $\sim 30 \mathrm{~km} \mathrm{~s}^{-1}$ (Mach number $\mathcal{M} \simeq 3$ for a $10^{4} \mathrm{~K}$ gas), which is the typical maximum velocity reached for champagne flows (TenorioTagle 1979). Therefore, the ionized gas will not accelerate much further (also reflected by the flattening of the emission profile behind $r_{\star}$; Figure 2 ), and the density in the flow remains roughly similar up until the shock front, i.e., $\rho_{1} \simeq 3.5 \mathrm{~cm}^{-3}$. Then the preshock density $\rho_{0}$ in which the shock front is moving can be determined through $\rho_{1} / \mathcal{M}^{2}=\rho_{0}$ (Bedijn \& Tenorio-Tagle 1981), from which we infer $\rho_{0}=0.4 \mathrm{~cm}^{-3}$, which is far below that observed for electron densities within evolved Galactic $H_{\text {II }}$ regions of similar size $\left(\gtrsim 50 \mathrm{~cm}^{-3}\right.$; Paladini et al. 2012), but typical for densities observed within the Warm Ionized Medium (WIM; Tielens 2005). Moreover, the existence of the cometary clouds L1617 and L1622, which are located behind Barnard's Loop as measured from the Orion $\mathrm{OB}$ association and have sharp I-fronts on their sides facing the Belt stars (Bally et al. 2009), reveals that the ionizing flux of the Belt stars must escape the GS206-17+13 shell, from which we conclude that the shell is incomplete and the bubble has likely "burst." Hence, we posit that the bubble interior has been emptied in its past and its contents were channeled into the Orion-Eridanus superbubble cavity.

The Orion Nebula is a prototypical blister $\mathrm{H}$ II region created by the young stars of the Trapezium $(<1$ Myr; Hillenbrand 1997; Bally 2008), with $\theta^{1}$ Ori $\mathrm{C}$ as the dominant ionizing source. The ionized gas is streaming from the near side of the OMC at about $10 \mathrm{~km} \mathrm{~s}^{-1}$ (O'Dell 2001) and accelerates into the surrounding medium. Recent velocity measurements of $\theta^{1}$ Ori $\mathrm{C}$ reveal that the star may be moving away from the OMC at a velocity of $13 \mathrm{~km} \mathrm{~s}^{-1}$ (see O'Dell et al. 2009, and references therein), which would imply a dynamical timescale of the Orion Nebula of only $1.5 \times 10^{4} \mathrm{yr}$, since the star is currently located $\sim 0.2 \mathrm{pc}$ away from the ionization front (IF). Indeed, the measured emission measure (EM) reveals that the thickness of the ionized gas layer is only 0.13 pc (Wen \& O'Dell 1995) assuming a constant density, which is known to be $\sim 6 \times 10^{3} \mathrm{~cm}^{-3}$ at the IF (O'Dell 2001). $\mathrm{X}$-ray emission from the $\sim 10^{6} \mathrm{~K}$ gas of the shocked stellar wind of $\theta$ Ori $\mathrm{C}$ (Güdel et al. 2008) has been detected in the Extended Orion Nebula (EON) and confirms the leakage of the (hot) gas toward the southwest, with the Orion-Eridanus superbubble as the most likely outlet of the flow of material.

\section{DISCUSSION}

Cowie et al. (1979) interpreted neutral absorption lines at $\left|v_{\mathrm{LSR}}\right| \lesssim 20 \mathrm{~km} \mathrm{~s}^{-1}$ toward stars in Orion as a thick, dense shell of swept-up material, partially ionized on the inside by the
Orion OB association. This ionized component would be evident as the IV gas and Barnard's Loop. However, the LV gas and IV gas are clearly separated in velocity space (Welty et al. 2002) toward $\zeta$ Ori at $(l, b)=(206.5,-16.6)$. This velocity separation makes it unlikely that LV and IV trace the same component of the Orion-Eridanus superbubble. In this respect, Bally (2008) already noted that the velocity dispersion of the ensemble of atomic and molecular clouds in Orion ranges over $>20 \mathrm{~km} \mathrm{~s}^{-1}$. Therefore, we attribute the lowvelocity neutral gas to clouds in the Orion region instead of a dense, neutral shell surrounding Orion. In addition, we have revealed the absence of a dense neutral shell surrounding Barnard's Loop (Section 3.2) and have shown that the Loop is part of a (separate) complete shell structure that is spatially correlated with HV gas detected toward Orion (Cowie et al. 1979; Welty et al. 2002), as discussed in Section 3.3.

The above-described results call for a thorough revisit of the structure, history, and subsequent evolution of the OrionEridanus superbubble. In Section 4.1.1 we connect the different gas tracers to the components of the superbubble and combine this with our observational work in order to derive a new and improved picture of the Orion-Eridanus region in Sections 4.1.2 and 4.1.3. We relate our findings to the stellar content of Orion OB1 in Section 4.2 and present a scenario for the future evolution of the Orion-Eridanus superbubble in Section 4.3.

\subsection{Connecting the Dots: An Updated Picture of the Orion-Eridanus Superbubble}

\subsubsection{Probing the Superbubble through Absorption Lines}

IV gas components are detected over a wide wavelength range, i.e., $-80 \mathrm{~km} \mathrm{~s}^{-1} \lesssim v_{\mathrm{LSR}} \lesssim-20 \mathrm{~km} \mathrm{~s}^{-1}$ (Cowie \& York 1978; Cowie et al. 1979; Huang et al. 1995; Welty et al. 2002). However, with high spectral resolution, high signal-to-noise ratio, and broad spectral coverage data, Welty et al. (2002) were able to locate the bulk of the IV gas toward $\zeta$ Ori in two single components at $v_{\mathrm{lsr}}=-35.3 \mathrm{~km} \mathrm{~s}^{-1}$ and $v_{\mathrm{lsr}}$ $=-31.6 \mathrm{~km} \mathrm{~s}^{-1}$.

We link the IV gas to the outer superbubble wall through several observations. First, the bulk of the IV gas lies within the velocity space reported for the superbubble wall in $\mathrm{HI}_{\mathrm{I}}$ $\left(-40 \mathrm{~km} \mathrm{~s}^{-1} \lesssim v_{\mathrm{LSR}} \lesssim 40 \mathrm{~km} \mathrm{~s}^{-1}\right.$; Brown et al. 1995). We note that by connecting the (ionized) IV to the superbubble wall, we assume that the outer wall toward $\zeta$ Ori is predominantly ionized along the line of sight. This might connect with our result that the eastern part of the superbubble wall does not exhibit a neutral shell, as was observed in region 1 of Figure 1 and will be further discussed in Section 4.1.3. Second, the total $\mathrm{H}$ II column density $\left(\log \left[N\left(\mathrm{H}_{\mathrm{II}}\right)\right]=18.44 \mathrm{~cm}^{-2}\right)$ and the postshock conditions of the IV gas toward $\zeta$ Ori $\left(n_{1}\right.$ $=0.16 \mathrm{~cm}^{-3}, T_{1}=9 \mathrm{e} 3 \mathrm{~K}$; Welty et al. 2002) imply an ionized shell thickness of $\sim 5.6 \mathrm{pc}$, in close agreement with the measured thickness of $1^{\circ}$ or $\sim 7 \mathrm{pc}$ of the $\mathrm{H} \alpha$ filaments along regions 1-5 in Figure 1. Third, the IV gas is widespread, extending well outside Barnard's Loop and encompassing the $\lambda$ Ori region, tracing a distinct component over an area larger than the HV gas (Section 3.3.1). Even though the definite extent of the IV gas should be established through an absorption-line study that includes sightlines covering the entire region shown in Figure 4, here we argue that that the IV gas traces a structure significantly larger than the dimensions of 
Table 2

The Connection between Observed Gas Velocities and Components within the Orion-Eridanus Region

\begin{tabular}{|c|c|c|c|c|c|c|c|}
\hline Component & $\begin{array}{c}v_{\mathrm{LSR}} \\
\left(\mathrm{km} \mathrm{s}^{-1}\right)\end{array}$ & $\begin{array}{c}n_{0} \\
\left(\mathrm{~cm}^{-3}\right)\end{array}$ & $\begin{array}{c}n_{1} \\
\left(\mathrm{~cm}^{-3}\right)\end{array}$ & $\begin{array}{c}\log \left[N_{\mathrm{H}}\right] \\
\left(\mathrm{cm}^{-2}\right)\end{array}$ & Ion. State & Reference & Tracing \\
\hline Low-velocity (LV) & -20 to 20 & $\ldots$ & $\ldots$ & 20.43 & neutral & 1 & Atomic/molecular clouds in Orion \\
\hline Intermediate-velocity (IV) & -80 to -20 & $\ldots$ & 0.16 & 18.44 & ionized & $1,2,3,4$ & Orion-Eridanus superbubble wall \\
\hline High-velocity (HV) & -120 to -80 & $3 \times 10^{-3}$ & $0.1-0.2$ & 17.88 & ionized & 1,2 & Nested shells (Barnard's Loop bubble, $\lambda$ Ori) \\
\hline
\end{tabular}

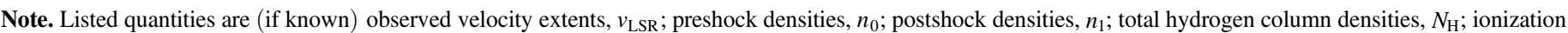

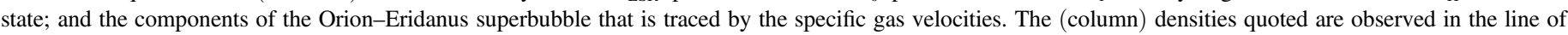
sight toward $\zeta$ Ori.

References. (1) Welty et al. (2002); (2) Cowie et al. (1979); (3) Cowie \& York (1978); (4) Huang et al. (1995).

the Orion-Eridanus superbubble described in previous works (Reynolds \& Ogden 1979; Heiles et al. 1999; Bally 2008; Pon et al. 2014b).

The above results reveal that the IV gas may be linked to the outer superbubble wall, whose neutral component was defined in Brown et al. (1995). In Section 3.3, we have argued that the HV gas traces the fast expansion of the Barnard's Loop bubble through the correlation between the sightlines that exhibit HV features and the morphology of the Barnard's Loop bubble from $\mathrm{H} \alpha$ (Figure 4). A similar result was obtained for the $\lambda$ Ori bubble. Both the Barnard's Loop bubble and the $\lambda$ Ori bubble may be connected to recent SN explosions (Sections 3.3.2 and 3.3.3) that could be at the origin of the HV gas. Finally, the LV gas is attributed to the ensemble of clouds in Orion (Bally 2008). As a caveat, we note that these conclusions are based on the study of a limited amount of sightlines that may not fully reflect the complexity of the Orion region (see Section 4.1.4). Nonetheless, based on the currently available data, we hypothesize that the different gas tracers trace separate components of the superbubble, summarized in Table 2.

\subsubsection{Extent of the Outer Superbubble Wall}

The extent and connection of the IV gas to the superbubble wall (Section 4.1.1) imply that the size of the Orion-Eridanus superbubble may be larger than previously thought. Indeed, Barnard's Loop is part of a separate closed bubble structure, suggesting that it is not associated with the large-scale expanding superbubble structure defined in earlier works (e.g., Reynolds \& Ogden 1979; Heiles et al. 1999; Bally 2008; Pon et al. 2014b). Here we argue that the outer $\mathrm{H} \alpha$ filament toward the southeast in Figure 1 offers a prime candidate for representing the outer superbubble wall.

We have concluded that Barnard's Loop is completely ionized by Orion $\mathrm{OB} 1$ and that ionizing photons are able to penetrate through. Moreover, in Sections 3.2.1 and 3.2.2, we have shown that Orion OB1 can provide the necessary ionizing photons to illuminate the $\mathrm{H} \alpha$ emission seen in Figure 1, indicating that the IF lies well outside the previously defined bounds of the Orion-Eridanus superbubble, which may be possible for superbubble walls in general (Basu et al. 1999). However, the large-scale, coherent $\mathrm{H} \alpha$ filament to the southeast is indicative of a limb-brightened shell, rather than a mere haphazard ISM structure that happened to be illuminated by ionizing photons piercing through Barnard's Loop.

We note that often the extent of superbubbles is traced through diffuse $\mathrm{X}$-ray emission. The photoelectric cross section for $0.25 \mathrm{keV}$ photons is $\sim 4 \times 10^{-21} \mathrm{~cm}^{2} \mathrm{H}^{-1}$ (Morrison \& McCammon 1983). By integrating the total foreground emission from the LAB survey (Kalberla et al. 2005), i.e.,
$-400<v_{\mathrm{LSR}}<0 \mathrm{~km} \mathrm{~s}^{-1}$ (which includes the near side of the superbubble shell at velocities $-40<v_{\mathrm{LSR}}<0 \mathrm{~km} \mathrm{~s}^{-1}$; Brown et al. 1995), we infer that the $0.25 \mathrm{keV}$ emission is confined to foreground $\mathrm{H}_{\mathrm{I}}$ column densities of $N_{\mathrm{H}} \lesssim 3 \times 10^{19} \mathrm{~cm}^{-2}$ (the superbubble cavity), where the optical depth for $0.25 \mathrm{keV} \mathrm{X}$ ray photons is $\tau_{\mathrm{x}} \lesssim 0.1$, while emission at $0.25 \mathrm{keV}$ is absent from regions of $N_{\mathrm{H}} \gtrsim 2 \times 10^{20} \mathrm{~cm}^{-2}$ (e.g., the east side of the superbubble near the Galactic plane, the northwest side of the superbubble near the high-lattitude clouds, and the superbubble wall in the west), where $\tau_{\mathrm{x}} \gtrsim 0.6$. Thus, the clear anticorrelation of the 0.25 (and 0.75$) \mathrm{keV}$ emission (Figure 1) with foreground $\mathrm{H}$ I limits the use of this tracer for many parts of the Orion-Eridanus region, especially toward the Galactic plane.

The association of Barnard's Loop with a closed separate bubble, the IV gas that traces a region larger than the previously defined bounds of the Orion-Eridanus superbubble, and the mere presence of the large-scale, coherent $\mathrm{H} \alpha$ filament to the southeast have led us to believe that regions 1-5 (Figure 3) trace the actual outer shell of the Orion-Eridanus superbubble. Projected in the interior of the outer shell are the Barnard's Loop bubble, the $\lambda$ Ori bubble, and the smaller-scale expanding bubbles discussed in Section 3.3.4. This indicates that the superbubble in its entirety consists of separate structures, or successive nested shells, possibly connected to a series of SN explosions originating from Orion OB1 over the past 15 Myr (Bally 2008; Sections 4.1.4 and 4.3.2). While hot plasma could exist between the $\mathrm{H} \alpha$ filament and Barnard's loop, the detection of the accompanying X-ray emission is hampered because of foreground absorption. Our hypothesis sprouted from studying the energetics of gas and dust (Sections 3.2.1 and 3.2.2) and their morphological appearances (Figure 1), combined with the connections of the different gas tracers with the components of the superbubble (Table 2). In the next subsection we discuss the temperature of the superbubble interior gas, as well as the global structure of the outer wall, in order to substantiate our claim regarding the structure and extent of the Orion-Eridanus superbubble.

\subsubsection{Temperature of the Superbubble Interior and Structure of the Outer Wall}

The evidence discussed in Section 4.1.2 indicates that the outer superbubble wall lies outside of Barnard's Loop (Section 4.1.2), possibly extending toward the outer $\mathrm{H} \alpha$ filament to the southeast (Figure 1). Here we quantify the temperature structure of the superbubble interior. First, we derive that the Barnard's Loop bubble is moving supersonically toward the east, from which we conclude that the temperature in this direction cannot be as high as $\gtrsim 10^{6} \mathrm{~K}$. After comparison with the cooling timescale for a gas at $<10^{6} \mathrm{~K}$, 
we infer that the medium between Barnard's Loop and the outer $\mathrm{H} \alpha$ filament must have cooled to $\sim 10^{4} \mathrm{~K}$, appropriate for a gas photoionized by Orion OB1. We argue that mass loading may cause temperature gradients within superbubble interiors, place our findings in the context of superbubble evolution, and discuss the structure of the outer wall.

The temperature structure of the superbubble interior can be estimated as follows. First, we define the isothermal sound speed, $c_{\mathrm{s}}=\left(k T / \mu m_{\mathrm{H}}\right)^{1 / 2}$, where $\mu$ is the mean mass per hydrogen nucleus $(=0.61$ for a fully ionized medium $)$. Toward the west of the Barnard's Loop bubble, the temperature of the superbubble interior traced by the X-ray-emitting gas is estimated at $T_{\mathrm{x}}=2.1 \times 10^{6} \mathrm{~K}$ (Burrows et al. 1993), giving $c_{\mathrm{s}}=170 \mathrm{~km} \mathrm{~s}^{-1}$. We compare this with the Barnard's Loop bubble expanding at $\sim 100 \mathrm{~km} \mathrm{~s}^{-1}$ (Section 3.3.2), which implies that the remnant of the recent $\mathrm{SN}$ explosion (Sections 3.3.2 and 4.3.1) is well within the radiative expansion phase (see below). During this phase, the velocity of the SNR decelerates with time as $\propto t^{-5 / 7}$ (Tielens 2005) if we assume that the ambient density in which the SNR propagates is distributed homogeneously. Adopting this timevelocity relation, the adiabatic expansion phase $\left(v_{\exp } \gtrsim\right.$ $250 \mathrm{~km} \mathrm{~s}^{-1}$ ) ended just below $10^{5} \mathrm{yr}$, and the bright crescent of Barnard's Loop has been moving subsonically for $\sim 0.2 \mathrm{Myr}$ (in case $c_{\mathrm{s}}=170 \mathrm{~km} \mathrm{~s}^{-1}$ ). When moving subsonically, sound waves will travel ahead of the shell and the structure would dissolve and merge with the ISM within a fadeaway timescale of (McKee \& Ostriker 1977; Draine 2011)

$$
\tau_{\text {fade }}=1.87 E_{51}^{0.32} n^{-0.37}\left(\frac{c_{\mathrm{s}}}{10 \mathrm{~km} \mathrm{~s}^{-1}}\right)^{-7 / 5} \mathrm{Myr},
$$

where $E_{51}$ is the kinetic energy in numbers of $10^{51} \mathrm{erg}$, and $n$ is the ambient hydrogen number density. The current kinetic energy of the eastern half of the Barnard's Loop bubble measures $E_{51} \sim 0.34$ (Section 4.3.1), part of which may already have been thermalized (Mac Low \& McCray 1988). However, the total kinetic energy delivered by an SN explosion typically does not exceed $E_{51} \sim 1$ (Veilleux et al. 2005), or $E_{51}$ $\sim 0.5$ for the eastern half of the bubble. With $E_{51}=0.5$ and parameters for the X-ray-emitting gas of the superbubble interior toward the west $\left(T_{\mathrm{x}}=2.1 \times 10^{6} \mathrm{~K}, n_{\mathrm{x}}=6 \times 10^{-3} \mathrm{~cm}^{-3}\right.$; Burrows et al. 1993; Section 4.3.1), we calculate $\tau_{\text {fade }} \sim$ 0.2 Myr, similar to the time Barnard's Loop has been moving subsonically in case it is moving within hot gas at a temperature exceeding $10^{6} \mathrm{~K}$.

The above-derived fadeaway timescale reveals that if Barnard's Loop would be moving to the east in an X-rayemitting gas such as that traced by ROSAT in the west part of the superbubble (Figure 1), it must be long in the process of merging and dissolving with the hot gas. This actual process may cause the faintness of the $\mathrm{H} \alpha$ emission of the Barnard's Loop bubble toward the west (Section 3.3.2), where the superbubble interior temperature is known to be at a temperature of $T_{\mathrm{x}}=2.1 \times 10^{6} \mathrm{~K}$ (Burrows et al. 1993; Figure 1). Clearly, this does not affect the eastern part of the Barnard's Loop bubble in the same way, as exemplified by the bright crescent of Barnard's Loop. Alternatively, there may be a density gradient within the superbubble interior that may cause the brightness difference of the Barnard's Loop bubble between its east and west sides. Either way, the conclusion is that the Barnard's Loop bubble moves supersonically toward the east, in line with the findings of Cowie et al. (1979), who argued that the HV gas (associated with the near side of the Barnard's Loop bubble; Section 3.3.2) traces a radiative shock. Consequently, the interior superbubble temperature toward the east cannot be at $\gtrsim 10^{6} \mathrm{~K}$, as is observed for the X-ray-emitting gas toward the west.

Temperature differences within a superbubble interior can originate from thermal conduction and evaporation of the swept-up superbubble shell. This mechanism may cool the interior of the superbubble by increasing the interior density (Weaver et al. 1977; Mac Low \& McCray 1988) such that radiative losses become important. Champagne flows and photoablation of molecular clouds enclosed in the superbubble can inject mass into the hot plasma, further lowering the temperature compared to that predicted by idealized models (a full discussion on the mass-loading mechanisms is deferred to Section 4.3). Gas below temperatures of $10^{6} \mathrm{~K}$ cools rapidly. At an interior density of $n_{\mathrm{x}}=6 \times 10^{-3} \mathrm{~cm}^{-3}$ (Burrows et al. 1993), the cooling timescale for a gas at $10^{5} \mathrm{~K}$ equals $\tau_{\text {cool }}$ $\sim k T / n \Lambda(T) \sim 10^{5} \mathrm{yr}$, where $\Lambda(T) \sim 5 \times 10^{-22} \mathrm{erg} \mathrm{cm}^{3} \mathrm{~s}^{-1}$ is the average value of the cooling function for gas at temperatures $10^{4} \mathrm{~K} \lesssim T \lesssim 10^{6} \mathrm{~K}$ (Dalgarno \& McCray 1972). Mass loading of the interior will shorten the cooling timescale. The detection of $\mathrm{Nv}$ gas (Welty et al. 2002) toward $\zeta$ Ori traces $\sim 10^{5} \mathrm{~K}$ gas and confirms the presence of a thermally conductive layer (Chu 2008), providing information on the temperature structure of the superbubble wall and the interior temperature, but it is unclear whether this conductive layer traces the outer superbubble wall or the interior shell associated with the Barnard's Loop bubble. Furthermore, it is uncertain whether this layer extends toward the outer $\mathrm{H} \alpha$ filament given the lack of coverage of absorption-line studies in this region (Figure 4). In any case, the cooling timescale is short compared to that of the estimated age of the superbubble $(\leqslant 8 \mathrm{Myr}$; Brown et al. 1994) and the SN rate in Orion OB1 (1-1.5 $\mathrm{Myr}^{-1}$; Bally 2008; Section 4.3.2) that may reheat the interior gas (see below and Section 4.3). Consequently, if the temperature drops below $10^{6} \mathrm{~K}$, the medium between Barnard's Loop and the outer filament will settle at a temperature of $\sim 10^{4} \mathrm{~K}$, as it is kept photoionized by Orion OB1 by photons piercing through Barnard's Loop (Section 3.2.2). Depending on the internal density, the medium between Barnard's Loop and the outer filament may not be in pressure equilibrium with the (higherdensity) outer $\mathrm{H} \alpha$ filament.

In the early stages of its evolution, an outer superbubble wall is defined by a shell of swept-up material, the expansion of which is driven by an overpressurized interior medium (Tielens 2005). The superbubble initially expands through the Sedov-Taylor solution, but as the interior medium expands adiabatically, its pressure will decrease and the expansion slows down. The radiative phase commences when the expansion velocity decreases to $\sim 250 \mathrm{~km} \mathrm{~s}^{-1}$ or a postshock temperature of $\sim 10^{6} \mathrm{~K}$ (Tielens 2005). At this point, radiative losses will become important, and the interior temperature (and pressure) will further drop. When the interior pressure reaches that of the ambient medium, the expansion of the superbubble wall will coast at constant radial momentum, thus slowing down as it sweeps up material from its surroundings. When the expansion velocity reaches the local sound speed, the superbubble wall will disappear and merge with the ISM within a fadeaway timescale (Equation (5)). The interior pressure may even drop below that of the ambient medium if the cooling 
timescale $\left(\sim 10^{5} \mathrm{yr}\right)$ is shorter than the pressure timescale or sound-crossing time. Currently, the distance between Barnard's Loop and the outer $\mathrm{H} \alpha$ filament is roughly $\sim 100 \mathrm{pc}$, such that the pressure timescale can be anywhere up to $\sim 10 \mathrm{Myr}$ for a $10^{4} \mathrm{~K}$ medium, depending on the exact moment the region cooled toward this temperature. However, the medium between Barnard's Loop and the outer filament must have cooled within the time between successive expanding shells (regulated by the $\mathrm{SN}$ rate, 1-1.5 $\mathrm{Myr}^{-1}$; Bally 2008). Thus, it may well be that the pressure inside the interior cannot adjust rapidly enough to the change in temperature and, in case no mass loading occurs, it can reach a lower pressure compared to the ambient galactic medium. In the case of an underpressurized interior, the expansion of the superbubble wall may halt and even reverse its movement if the pressure difference between the interior and the ambient medium is strong enough.

Given that the medium between Barnard's Loop and the outer $\mathrm{H} \alpha$ filament is at $10^{4} \mathrm{~K}$ and we are not able to constrain its density, one may question whether this medium in fact represents the WIM or the Warm Neutral Medium (WNM; Tielens 2005). In this case, the outer $\mathrm{H} \alpha$ filament is not the superbubble wall, but merely an ISM feature illuminated by photons escaping from Barnard's Loop. However, we have revealed that Barnard's Loop is part of a separate closed structure (Section 3.3.2), and we have argued that the superbubble wall extends beyond Barnard's Loop (addressed in Sections 4.1.1 and 4.1.2). Surely, if one accepts that Barnard's Loop is expanding at $100 \mathrm{~km} \mathrm{~s}^{-1}$ and represents an SNR of age 0.3 Myr (Sections 3.3.2 and 4.3.1), it may not be surprising that the superbubble wall lies further outward as measured from Orion OB1, given that star formation has occurred over $15 \mathrm{Myr}$ in Orion and that 10-20 SNe have gone off in its past (Bally 2008). Here we argue that successive SN explosions may lead to the appearance of successive shells. In this scenario, the large-scale, coherent $\mathrm{H} \alpha$ filament to the southeast represents a prime candidate to trace the actual outer superbubble wall. We defer a further discussion on the idea of successive shells and the evolution of the outer superbubble wall to Section 4.3.2.

We note that the outer $\mathrm{H} \alpha$ filament (Figure 1) does not contain a neutral shell that is expected for a sweeping superbubble wall (Figures 2 and 3 ). The lack of a neutral shell may simply suggest that the swept-up ISM did not contain enough material to fully absorb the ionizing photons from Orion OB1. Alternatively, the east side of the superbubble, which is racing up the density ramp toward the Galactic plane, may not be moving supersonically anymore and has changed character from a shock wave to a sound wave, rendering it unable to sweep up additional mass. Using Equation (5) and $E_{51} \sim 10$ (the total kinetic energy exerted by Orion OB1; Brown et al. 1995; Bally 2008), we calculate for the east side of the bubble expanding toward the WNM of the Galactic plane $\left(n \sim 0.5 \mathrm{~cm}^{-3}, c_{\mathrm{s}} \sim 10 \mathrm{~km} \mathrm{~s}^{-1}\right) \tau_{\text {fade }} \simeq 4 \mathrm{Myr}$, well below the estimated age of the superbubble ( $\lesssim 8$ Myr; Brown et al. 1994). Thus, we may be observing the east side of the superbubble as it is in the process of dissolving, already having lost its neutral shell and currently being completely ionized before it merges with the local material of the ISM. Equation (5) does not apply to the west side of the superbubble as its expansion velocity (traced through $\mathrm{H}$, $\sim 40 \mathrm{~km} \mathrm{~s}^{-1}$; Brown et al. 1995) implies that it moves supersonically through the WNM/WIM. However, for the ionized east side of the superbubble the expansion

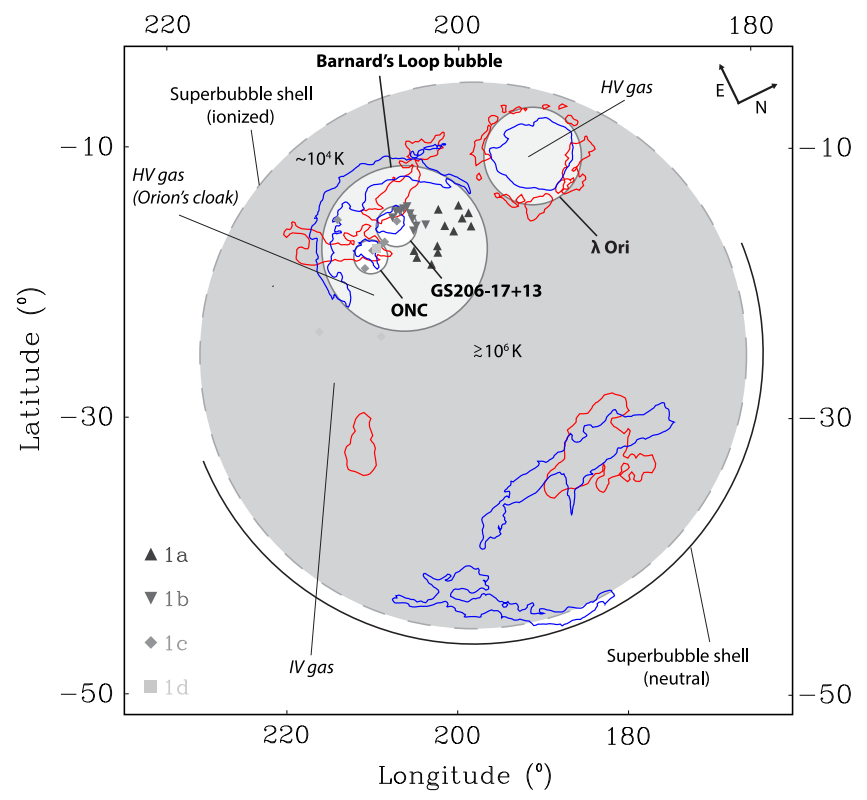

Figure 6. Schematic of the Orion-Eridanus superbubble and several of its major components. See Figure 1 for the nomenclatures of the shown structures. The proposed outer shell of the Orion-Eridanus superbubble (dashed circle), blown by a series of $\mathrm{SNe}$ from an old subpopulation of the Orion $\mathrm{OB}$ association, is traced by an intermediate-velocity (IV) shock that sweeps up the ambient ISM (gray area). Toward the west, the superbubble is surrounded by a shell of neutral (solid black line) swept-up material, whereas this shell is completely ionized (dashed gray line) toward the east. The temperature in the superbubble interior seems to be lower toward the east $\left(\sim 10^{4} \mathrm{~K}\right)$ in the direction of the Galactic plane, compared to the X-ray-emitting gas in the west $\left(\geq 10^{6} \mathrm{~K}\right)$. The outer shell encompasses several "nested" smaller shells or bubbles (solid gray circles, denoted in boldface) that are more recently formed, such as the Barnard's Loop bubble, traced by high-velocity (HV) gas. Other examples of bubbles that may have been triggered by ongoing activity in Orion OB1 are the $\lambda$ Ori bubble, GS206-17+13, and the shell surrounding the Orion Nebula Cluster (ONC).

velocity is not constrained, and the question whether or not it is moving supersonically remains unanswered.

\subsubsection{Summary: The Structure of the Orion-Eridanus Superbubble}

We now have obtained the complete set of tools to derive an updated picture of the Orion-Eridanus region, which we show in Figure 6. Our results demonstrate that the superbubble consists of separate structures or nested shells, superimposed along the line of sight. In particular, we have recognized a separate bubble (among others; Section 3.3), evident as the HV gas and the Barnard's Loop bubble in $\mathrm{H} \alpha$ (Figure 4). This bubble expands inside the preexisting cavity of the OrionEridanus superbubble, given its preshock density, its embedded nature in the Orion-Eridanus region (Figure 6), and its likely origin through feedback from Orion OB1. The realization that Barnard's Loop is not part of the superbubble wall (Section 3.3.2), the IV gas that seems to trace a region larger than the previously defined bounds of the Orion-Eridanus superbubble (Section 4.1.1), and the presence of the large-scale, coherent $\mathrm{H} \alpha$ filament to the southeast have led us to believe that the extent of the Orion-Eridanus superbubble is larger than previously throught. We have argued that the temperature of the superbubble interior between Barnard's Loop and the outer $\mathrm{H} \alpha$ filament to the southeast cannot be as high as that observed for the X-ray-emitting gas toward the west (Burrows et al. 1993) and therefore must have settled at $10^{4} \mathrm{~K}$ 
(Section 4.1.3). The expansion of this outer $\mathrm{H} \alpha$ filament may not be pressure driven anymore. While this is an inevitable outcome during late stages of superbubbles and SNRs (Tielens 2005), the interior pressure drop may be accelerated through the effects of mass loading and radiative energy losses (Section 4.3). The observations (Figure 1) indicate that the outer superbubble wall is surrounded by a shell of $\mathrm{H}$ i toward the west, whereas it may be completely ionized toward the east (Section 4.1.3).

We stress that the association of gas velocities with components in the superbubble (Table 2) is largely based on the analysis of a dozen sightlines throughout the Orion side of the superbubble (Figure 4). It is not clear how much mass is associated with these velocities (Section 4.3.1). Moreover, some of the gas tracers are not seen in particular lines of sight. This may be because we are tracing foreground stars, such as the sightline toward $\gamma$ Ori located at 77 pc (van Leeuwen 2007), which could provide a constraint to the elongation of the bubble toward the Local Bubble (e.g., Bally 2008; Pon et al. 2014b), or incomplete spectral coverage (Cowie et al. 1979). Alternatively, the distribution of the gas components might be patchy (Cowie et al. 1979). Surely, the connections laid out in Table 2 may be oversimplified, as the Orion region is highly complex, containing dozens of filamentary clouds, and must bear the marks of multiple SNRs (Bally 2008) that are breaking out of a region that contains a lot of substructure. Further observations are needed to firmly constrain the extent of the distinct gas components in order to derive the detailed structure of the Orion-Eridanus superbubble and to recognize the existence of conductive layers that would provide more insight in the temperature distribution throughout the superbubble interior.

The observations of the gas and dust have revealed that Orion OB1 can be the source of ionization of the $\mathrm{H} \alpha$ filaments encompassed within the entire region shown in Figure 1 (Section 3.2). We have connected the IV gas with the outer superbubble wall. We note that the preshock density of the IV is not known. However, given a postshock density of $0.16 \mathrm{~cm}^{-3}$ (Welty et al. 2002) and a shock velocity of $40 \mathrm{~km} \mathrm{~s}^{-1}$ (Brown et al. 1995), a shock moving through the WNM $(T=8000 \mathrm{~K}$, $c_{\mathrm{s}} \sim 10 \mathrm{~km}^{-1}$ ) would have a preshock density of order $\sim 0.01 \mathrm{~cm}^{-3}$ (J-type shocks increase densities by $\mathcal{M}^{2}$; Tielens 2005), which is somewhat low for the WNM in the Galactic plane. Higher densities for the Orion-Eridanus shell of $1-5 \mathrm{~cm}^{-3}$ are quoted toward the Eridanus filaments in the west (e.g., Reynolds \& Ogden 1979), indicating that the expansion occurs somewhat anisotropically, which can also be inferred by appreciating the complex morphology of the superbubble. In any case, the shock velocity of the IV gas $\left(\sim 40 \mathrm{~km} \mathrm{~s}^{-1}\right)$ is not fast enough to ionize hydrogen; therefore, the Eridanus filaments (and the IV gas) are photoionized by Orion OB1, which was already noted by Reynolds \& Ogden (1979) and Welty et al. (2002). We note that Pon et al. (2014b) explored other mechanisms of ionization but concluded that Orion OB1 most likely causes the observed ionization state of the IV gas. When the spherical geometry from Figure 6 is adopted for the outer superbubble wall, and assuming a diameter of the supershell of $45^{\circ}$ (equivalent to a radius of $160 \mathrm{pc}$ ) with expansion velocity of $\sim 40 \mathrm{~km} \mathrm{~s}^{-1}$, the dynamical age of the shell is $\sim 4 \mathrm{Myr}$, consistent with the age of the Orion OB1a subgroup, minus a main-sequence lifetime of 3-5 Myr for its most massive stars (Bally 2008). Naturally, stellar winds during the first few Myr of the lifetime of the OB association may have contributed to the formation and expansion of the superbubble wall as well.

In conclusion, considering that star formation had been occurring for 10-15 Myr in Orion OB1 and the presence of at least four distinct subgroups (Blaauw 1964; Bally 2008), it may not be surprising that the Orion-Eridanus superbubble consists of a set of nested shells. Our results connect well with recent work from Schlafly et al. (2015), who show that the OMCs are in turn part of an ancient ring of dust. Possibly, the ring represents the remainder of a large bubble that pushed the OMCs out of the Galactic plane toward their current location. Still, the hierarchy of bubbles does not end at the possible progenitor of the Orion-Eridanus superbubble defined by Schlafly et al. (2015). Indeed, it has been argued that the Gould's Belt that includes all nearby OB associations is associated with a large expanding ancient (30-60 Myr) supershell known as Lindblad's ring (see Bally 2008, and references therein). The Gould's Belt of stars and OB associations may therefore represent sequential star formation when the Lindblad's ring cooled and collapsed to form dark clouds, out of which the evolution of the Orion region commenced.

\subsection{The Nested Shells and Their Relation to Orion OBI}

The recent discovery that the OMCs may be part of an ancient ring of dust (Schlafly et al. 2015), the uncovering of an extended foreground population toward Orion A (Alves \& Bouy 2012; Bouy et al. 2014), and the mysterious origin of some of the brightest stars in Orion ( $\alpha$ Ori, $\beta$ Ori, and $\kappa$ Ori; Bally 2008) illustrate that our current understanding of the Orion region is still limited. Bally (2008) proposed that the bright stars may be part of a foreground group about $150 \mathrm{pc}$ in front of Orion OB1 and together constitute the three most massive members of this population still present to date. Alternatively, some of the massive stars from Orion OB1 may have been ejected as runaways (space velocities in excess of $>40 \mathrm{~km} \mathrm{~s}^{-1}$ ) in the direction of the Local Bubble before exploding as SNe: up to $25 \%$ of the OB stars end up as runaways (Zinnecker \& Yorke 2007). Such stars could be linked to the odd geometry of the Orion-Eridanus superbubble (Pon et al. 2014b), which appears to be elongated toward the Local Bubble.

One must keep in mind that most of the distance determinations toward the Orion stellar populations are based on photometric indicators (e.g., Brown et al. 1994). Although the Hipparcos mission determined parallaxes for many stars in the Orion region, the distance to the young populations $(\sim 300-400 \mathrm{pc})$ translates to Hipparcos parallax errors of $30 \%-40 \%$ (given a precision of 1 mas; Perryman et al. 1997). Thus, the detailed distribution of the stellar population along the line of sight cannot, as of yet, be determined (see also de Zeeuw et al. 1999). In this respect, the Gaia mission (Perryman et al. 2001; de Bruijne 2012) is expected to dramatically improve the distance and age determinations of the stars and subgroups in Orion. The entire Orion region lies well within the range where Gaia parallaxes will be accurate to $10 \%$ or better over a large range of spectral types. This will allow us to constrain the relationship between the stellar content and the nested shells seen in the OrionEridanus superbubble and to address the importance of triggered star formation (Elmegreen \& Lada 1977) in Orion, 
for which one needs precise measurements of proper motion, radial velocities, and ages.

\subsection{Evolution of the Orion-Eridanus Superbubble}

In this section, we exploit the results drawn from the previous sections to derive an updated picture of the evolution of the Orion-Eridanus superbubble, regulated by a balance between processes that mass-load the superbubble interior, controlling its temperature and density structure (Cowie et al. 1981; Hartquist et al. 1986), and feedback from Orion OB1 that sweeps up and collects the injected interior mass in expanding shells before thermalizing or cooling (Mac Low \& McCray 1988). These processes determine the momentum of the superbubble wall and the thermal pressure of the interior, which drives the superbubble expansion out into the ambient Galactic medium.

First, in Section 4.3.1 we summarize masses and energetics as observed from different components of the Orion-Eridanus superbubble. From this, we conclude that the Barnard's Loop bubble is an SNR (associated with an SN explosion that occurred $3 \times 10^{5}$ yr ago; Section 3.3.1; Cowie et al. 1979), and we argue in Section 4.3.2 that the SNR has swept through the inner parts of the superbubble to collect mass that has been introduced through champagne flows and thermally evaporating clouds. Behind the Barnard's Loop bubble, champagne flows from IC 434, the Orion Nebula, and $\lambda$ Ori continue to mass-load the superbubble interior until the next SN explosion will start the cycle all over again. The net effect is a gradual disruption of the star-forming reservoir and the subsequent outward transportation of processed material, where it will ultimately pressurize the superbubble interior, or add momentum to the outer superbubble wall. We argue that at the current rate, the OMCs will be able to power the expansion and evolution of the Orion-Eridanus superbubble for another 20-30 Myr, before they are depleted and run out of steam, at which point the superbubble will disappear and merge with the surrounding ISM.

\subsubsection{Masses and Energetics of the Orion-Eridanus Superbubble}

Hot gas ( $\gtrsim 10^{6} \mathrm{~K}$; Table 3$)$ from the superbubble interior has been characterized by Burrows et al. (1993), who inferred a temperature of $T_{\mathrm{x}} \sim 2 \times 10^{6} \mathrm{~K}$ and a total luminosity at X-ray wavelengths of $L_{\mathrm{x}} \approx 8.0 \times 10^{35} \mathrm{erg} \mathrm{s}^{-1}$, when assuming that the $\mathrm{X}$-ray-emitting region is spherical and its actual "hook-shaped" appearance results after foreground absorption by MBM 18 and MBM 20 (Figure 1; Burrows et al. 1993). The authors deduce the following parameters for the hot gas: when $400 \mathrm{pc}$ is taken as the distance toward the superbubble, the radius of the X-rayemitting region is $r_{\mathrm{x}}=70 \mathrm{pc}$, its density is $n_{\mathrm{x}}=6 \times 10^{-3} \mathrm{~cm}^{-3}$ (Burrows et al. 1993), and it contains a total mass $M_{\mathrm{x}}=230$ $M_{\odot}$ after adopting a spherical geometry. However, if we adopt the (larger) geometry from Figure 6 and account for possible missing X-ray emission because of foreground absorption (Figure 1 and Section 4.1.2), the total mass within a region of $r_{\mathrm{x}}=160 \mathrm{pc}$ would amount to $M_{\mathrm{x}}=1800 M_{\odot}$. Table 3 also denotes a value for the hot gas inside the Barnard's Loop bubble of radius $35 \mathrm{pc}$ in case it were filled with gas of the same characteristics as observed in the superbubble. As noted before (Section 4.1.2), the high column densities for the Barnard's Loop region prohibit the detection of diffuse X-rays toward the east side of the Barnard's Loop bubble. Hot gas is detected toward the west side, where the $\mathrm{H}$ I foreground column is much lower, but it is unclear whether this emission traces hot gas from the Barnard's Loop bubble or the entire OrionEridanus superbubble.

Ionized gas $\left(\sim 10^{4} \mathrm{~K}\right.$; Table 3$)$ in the Orion-Eridanus region is associated with the outer $\mathrm{H} \alpha$ filaments of the superbubble, the Barnard's Loop bubble shell, and the champagne flows of the $\lambda$ Ori bubble, GS206-17+13, the IC 434 emission nebula, and the Orion Nebula (Sections 3.2 and 3.3). For Barnard's Loop, the total ionized mass is estimated at $M=6700 M_{\odot}$ by adopting a half-sphere geometry with thickness $1^{\circ}$, outer radius of $7^{\circ}$, and density $n_{\mathrm{H}}=3.4 \mathrm{~cm}^{-3}$ (Section 3.1 ). The western half of the complete bubble (Figure 4) is much fainter, which implies that the density in the Barnard's Loop bubble varies significantly between both parts (Section 4.1.3). Assuming $v_{\mathrm{exp}}$ $=100 \mathrm{~km} \mathrm{~s}^{-1}$ (Section 3.3), $E_{\text {kin }}=3.4 \times 10^{50} \mathrm{erg}$. The total energy that has been injected in the region can then be estimated at $E_{\text {kin }}=6.7 \times 10^{50} \mathrm{erg}$ by assuming spherical symmetry. This energy should be compared to the typical kinetic energy provided per SN $\left(10^{50}-10^{51} \mathrm{erg}\right.$, depending on the efficiency at which it couples to the ISM; Veilleux et al. 2005), as well as the wind mechanical energy input. By adopting typical wind parameters $\left(\dot{M}=1 \times 10^{-6} M_{\odot} \mathrm{yr}^{-1}, v_{\infty}\right.$ $=2000 \mathrm{~km} \mathrm{~s}^{-1}$ ) for the seven most massive stars in the Orion OB association and assuming the lifetime of Orion's Cloak at 3 $\times 10^{5} \mathrm{yr}$ (Section 3.3.1; Cowie et al. 1979), the total mechanical luminosity exerted by the Orion OB association is $L_{\text {mech }}$ $=8.4 \times 10^{49} \mathrm{erg}$, of which $\sim 20 \%$ couples to the surrounding medium as kinetic energy (van Buren 1986). Thus, the (combined efforts of) stellar winds from the Orion OB1 stars seem insufficient to provide the measured kinetic energy of the Barnard's Loop bubble, and we associate the Barnard's Loop bubble with a recent $\mathrm{SN}$ explosion.

Table 3 reflects the omnipresence of champagne flows in the Orion region, consistent with the findings of Ochsendorf et al. (2014b), who showed that $\mathrm{H}$ II region champagne flows are ubiquitous throughout the Galactic plane and illustrated the importance of this phase to the turbulent structure of the ISM. Still, the amount of material that is eroded from the molecular clouds and delivered by champagne flows in the form of kinetic energy of the fast-moving ionized gas is difficult to determine, and estimates over the lifetime of the star will depend most critically on the ionizing luminosity and main-sequence lifetime of the ionizing star (e.g., Whitworth 1979). Here we follow Tielens (2005) and adopt a "typical" blister phase that will accelerate $M_{\odot} \sim 3 \times 10^{3} M_{\odot}$ of ionized gas to $30 \mathrm{~km} \mathrm{~s}^{-1}$, providing a kinetic energy of $E_{\text {kin }} \sim 3 \times 10^{49} \mathrm{erg}$. We use this value for the champagne flow of the GS206-17+13 bubble that has occurred in the past (Section 3.3.4). For comparison, the ionized interior density of the $\lambda$ Ori region, which seems to be on the verge of breaking out (Section 3.3.3), is quoted to be between $2000 \quad M_{\odot} \quad\left(\right.$ van Buren 1986) and $6000 \quad M_{\odot}$ (Reich 1978); this would add an amount of mass and kinetic energy to the superbubble interior similar to that given by the GS206-17+13 shell. The Orion Nebula has just recently entered the champagne phase (Section 3.3.4) and only contains some $\sim 20 M_{\odot}$ of ionized gas (Wilson et al. 1997). The IC 434 region is a special case, as the champagne flow originates from a chance encounter between the $\sigma$ Ori star cluster and the Orion B molecular cloud (Ochsendorf et al. 2014a; Ochsendorf \& Tielens 2015). Still, the star has already eroded $100 M_{\odot}$ and will add another $350 M_{\odot}$ to the ionized gas before it plunges 
Table 3

Masses and Energetics of the Orion-Eridanus Superbubble

\begin{tabular}{|c|c|c|c|c|c|c|}
\hline Component & $\begin{array}{c}M \\
\left(10^{3} M_{\odot}\right)\end{array}$ & $\begin{array}{c}v_{\exp } \\
\left(\mathrm{km} \mathrm{s}^{-1}\right)\end{array}$ & $\begin{array}{c}E_{\mathrm{th}} \\
\left(10^{49} \mathrm{erg}\right)\end{array}$ & $\begin{array}{c}E_{\text {kin }} \\
\left(10^{49} \mathrm{erg}\right)\end{array}$ & Form & Reference \\
\hline \multicolumn{7}{|l|}{$\overline{\text { Hot gas }\left(\gtrsim 10^{6} \mathrm{~K}\right)}$} \\
\hline Superbubble interior & 1.8 & $\cdots$ & 62 & $\cdots$ & Thermal energy & Burrows et al. (1993) \\
\hline Barnard's Loop bubble interior & $(0.02)$ & $\cdots$ & $(0.65)$ & $\cdots$ & Thermal energy & This work \\
\hline \multicolumn{7}{|l|}{ Ionized gas $\left(10^{4} \mathrm{~K}\right)$} \\
\hline Superbubble & 84 & $15(1)$ & 14 & 19 & Swept-up shell & Reynolds \& Ogden (1979) \\
\hline Barnard's Loop bubble & 6.7 & 100 & 1 & 67 & Swept-up shell & This work \\
\hline$\lambda$ Ori & $2-6$ & 30 & 0.5 & 3 & Champagne flow & Reich (1978), van Buren (1986) \\
\hline GS206-17+13 & 3 & 30 & 0.5 & 3 & Champagne flow & This work \\
\hline IC 434 & 0.1 & 30 & 0.02 & 0.1 & Champagne flow & Ochsendorf \& Tielens (2015) \\
\hline Orion Nebula & 0.02 & 30 & 0.003 & 0.02 & Champagne flow & Wilson et al. (1997) \\
\hline \multicolumn{7}{|l|}{ Neutral gas $\left(10^{2} \mathrm{~K}\right)$} \\
\hline Superbubble & 250 & 40 & $\cdots$ & 370 & Swept-up shell & Brown et al. (1995) \\
\hline GS206-17+13 & 3.4 & 8 & $\cdots$ & $0.2(2)$ & Swept-up shell & Ochsendorf \& Tielens (2015) \\
\hline Orion Nebula (Veil) & 2.3 & 2 & $\cdots$ & 0.01 & Swept-up shell & van der Werf et al. (2013); this work \\
\hline \multicolumn{7}{|l|}{ Molecular gas $(10 \mathrm{~K})$} \\
\hline OMC A & 105 & $\cdots$ & $\cdots$ & $\cdots$ & Molecular cloud & Wilson et al. (2005) \\
\hline OMC B & 82 & $\cdots$ & $\cdots$ & $\cdots$ & Molecular cloud & Wilson et al. (2005) \\
\hline$\lambda$ Ori & 11 & 16.5 & $\cdots$ & 6 & Swept-up shell & Lang \& Masheder (1998) \\
\hline \multicolumn{7}{|l|}{ Stars } \\
\hline Ori 1a & 1.6 & $\ldots$ & $\cdots$ & $\cdots$ & Stellar cluster & Brown et al. (1994) \\
\hline Ori $1 b$ & 1.3 & $\ldots$ & $\cdots$ & $\cdots$ & Stellar cluster & Brown et al. (1994) \\
\hline Ori $1 \mathrm{c}$ & 1.8 & $\ldots$ & $\ldots$ & $\ldots$ & Stellar cluster & Brown et al. (1994) \\
\hline Ori 1d (ONC) & 1.8 & $\cdots$ & $\cdots$ & $\cdots$ & Stellar cluster & Hillenbrand (1997) \\
\hline Ori 1d (NGC2024) & 0.2 & $\cdots$ & $\cdots$ & $\cdots$ & Stellar cluster & Comeron et al. (1996) \\
\hline$\sigma$ Ori & 0.2 & $\ldots$ & $\cdots$ & $\ldots$ & Stellar cluster & Sherry et al. (2004) \\
\hline$(\lambda$ Ori $)$ & $(0.6)$ & $\cdots$ & $\cdots$ & $\cdots$ & Stellar cluster & Barrado y Navascués et al. (2004) \\
\hline
\end{tabular}

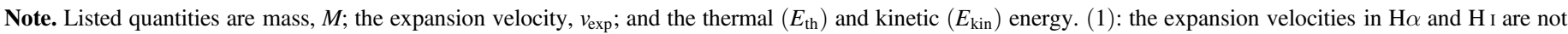

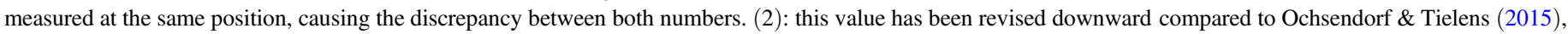

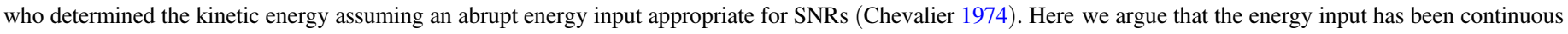
through stellar winds (see the text) and instead use $E_{\text {kin }}=1 / 2 M v_{\exp }^{2}$.

into the molecular cloud (for a current distance of $3.5 \mathrm{pc}$ and a closing velocity of $\sim 10 \mathrm{~km} \mathrm{~s}^{-1}$; Ochsendorf \& Tielens 2015$)$.

Neutral gas $\left(\sim 10^{2} \mathrm{~K}\right.$; Table 3$)$ is present in the superbubble wall (Brown et al. 1995) and the slowly expanding shells of the $\lambda$ Ori region (Lang \& Masheder 1998), the GS206-17+13 bubble (Ochsendorf \& Tielens 2015), and the Orion Nebula that is encapsulated by the neutral "Veil" (e.g., van der Werf et al. 2013). The GS206-17+13 is a stellar-wind-blown bubble (Section 3.3.4). The Orion Nebula Veil contains a total neutral column density of $N_{\mathrm{H}}=5.8 \times 10^{21} \mathrm{~cm}^{-2}$, has a size of at least $1.5 \mathrm{pc}$, as it is common to the Orion Nebula and M43, and shows only minor blueshifted velocities with respect to the background OMC $\left(\sim 2 \mathrm{~km} \mathrm{~s}^{-1}\right)$. This implies that the mechanical feedback of the Trapezium through, e.g., the champagne flow or stellar winds has not yet coupled as kinetic energy to the surrounding neutral gas, consistent with the young age of the Trapezium stars (<1 Myr; Hillenbrand 1997) and, perhaps, the very small dynamical time inferred for the Orion Nebula since the onset of the champagne flow $\left(\sim 1.5 \times 10^{4} \mathrm{yr}\right.$; O'Dell et al. 2009). Here we estimate the total $\mathrm{H}_{\mathrm{I}}$ mass for the Orion Veil by assuming that the measured column density by van der Werf et al. (2013) is homogeneously distributed over a shell with a radius of $2 \mathrm{pc}$, large enough to cover both the M43 region and the entire EON (Güdel et al. 2008).
Molecular gas ( $\sim 10 \mathrm{~K}$; Table 3$)$ is detected in dozens of small cometary clouds but largely concentrates in the OMCs, which constitute the main reservoir of the dense gas out of which star formation occurs. As described by, e.g., Bally (2008), the ages and locations of the subgroups in Orion OB1 indicate that star formation has propagated through the "protoOrion cloud" sequentially. The kinetic energy associated with the expansion of the $\lambda$ Ori molecular shell $\left(6 \times 10^{49} \mathrm{erg}\right)$ is consistent with expansion driven by either the overpressure of photoionized gas (Spitzer 1978), which deposits some $10^{49}$ $10^{50} \mathrm{erg}$ to the surrounding medium (Ochsendorf et al. 2014b) over the lifetime of $\lambda$ Ori ( $\sim 10 \mathrm{Myr})$, or an SN explosion (Veilleux et al. 2005), or a wind-blown origin (Weaver et al. 1977), as $L_{\text {mech }}=2 \times 10^{50} \mathrm{erg}$ for $\lambda$ Ori (van Buren 1986) assuming an age of $5 \mathrm{Myr}$ (Bally 2008), a fraction of which will couple as kinetic energy. Some previous works favor an $\mathrm{SN}$ origin for the $\lambda$ Ori bubble, which would also explain the current space motion of the $\lambda$ Ori star (see Section 3.3.3).

Stars are listed for comparison, where we have included the separate subgroups from the Orion OB1 association (1a, 1b, 1c, and 1d; Blaauw 1964; Brown et al. 1994). The masses in the $1 \mathrm{a}, 1 \mathrm{~b}$, and 1c subgroups have been determined using the results in Brown et al. (1994), who constructed the initial mass function (IMF) and through this reported the number of stars 
initially found in the 2-120 $M_{\odot}$ mass range for each subgroup. We complement this by estimating the contribution from lowmass stars below the completion limit of the Brown et al. (1994) study (0.08-2 $\left.M_{\odot}\right)$ using a standard IMF (Kroupa 2001), normalized to the results from Brown et al. (1994). Table 3 lists the resulting total masses, assuming an upper stellar mass limit set by the age of each subgroup. In Orion OB1a, stars more massive than $13 M_{\odot}$ have exploded as $\mathrm{SNe}$, while the (younger) age of the Orion $1 \mathrm{~b}$ and $1 \mathrm{c}$ subgroups implies an upper mass limit of about $20 M_{\odot}$ (Bally 2008). Orion OB1d is the youngest subgroup and includes many starforming clusters (Bally 2008), and here we only include the two largest components, the ONC in OMC A and NGC 2024 in OMC B. The $\sigma$ Ori group is noteworthy here, as it has traditionally been assigned to Orion OB $1 \mathrm{~b}$ based on its spatial location among the Belt stars, but its traversed space motion reveals that the cluster might have originated from the Orion OB1c region (Ochsendorf \& Tielens 2015). We have bracketed the $\lambda$ Ori region in Table 3 as it is separated from the star clusters that are associated with the OMCs and does not directly influence the scenario that will be outlined in Section 4.3.2.

Summary: most of the mass of the Orion-Eridanus superbubble resides in the outer superbubble wall, while the nested shells that process and sweep up the interior material (see Section 4.3.2) constitute only a small part. The molecular clouds still represent a large reservoir of mass, comparable to that located in the superbubble wall. Most of the energy is found in the form of kinetic energy of the expansion of the superbubble wall. Still, about $10 \%-15 \%$ of the total energy is located in thermal energy of the hot gas of the superbubble interior, and a comparable amount lies within the kinetic energy of the ionized gas. The thermal energy of the hot gas created by fast shocks is large compared to the thermal energy of the $10^{4} \mathrm{~K}$ ionized gas delivered through photoionization from Orion OB1. Finally, when all subgroups in Table 3 are combined, stars comprise some $\sim 6.9 \times 10^{3} M_{\odot}$, or $3 \%-4 \%$ of the molecular material within the OMCs.

\subsubsection{Mass Loading and Cleansing of the Interior}

The expanding shells in the interior of the Orion-Eridanus superbubble are the manifestation of feedback from Orion OB1. When moving supersonically, the shockwaves associated with the shells sweep up the interior mass of the superbubble, acting as a mechanism to remove any material that may have been introduced to the medium in the past. In this respect, the mass $\left(6.7 \times 10^{3} M_{\odot}\right)$ and radius $(35 \mathrm{pc})$ of the shell surrounding the Barnard's Loop bubble can be combined to show that the average density of the swept-up medium contained $\sim 1 \mathrm{~cm}^{-3}$. We note that the density of the swept-up material only slightly exceeds what would be expected for the WNM of the Galaxy, which would be consistent with the Barnard's Loop bubble being a superbubble wall expanding into the ambient Galactic medium. However, we render this scenario unlikely given the short dynamical timescale of the Barnard's Loop bubble ( $\sim 0.3 \mathrm{Myr}$; Section 3.3.2) compared to the Orion star-forming region ( $\sim 15 \mathrm{Myr}$; Bally 2008$)$, the preshock density of the $\mathrm{HV}$ gas $\left(\sim 3 \times 10^{-3} \mathrm{~cm}^{-3}\right.$; Welty et al. 2002), and its nested appearance within the entire OrionEridanus superbubble (Figures 1 and 4). The increased density is about two orders of magnitude above what is expected for a superbubble interior $\left(\sim 10^{-2} \mathrm{~cm}^{-3}\right.$; Weaver et al. 1977), which provides convincing evidence that the superbubble interior must have been mass-loaded since the last SN passed through and cleansed the interior.

Photoionization feedback from Orion OB1 ultimately creates champagne flows that channel $10^{4} \mathrm{~K}$ gas from the $\mathrm{H}$ II regions into the superbubble cavity, where it will mix with the hot gas $\left(\gtrsim 10^{6}\right.$; Chu 2008). The injected mass will significantly lower the temperature of the superbubble interior and increase its density. The total mass delivered to the interior by a typical champagne flow over the lifetime of a massive star $\left(\sim 3 \times 10^{3}\right.$ $\left.M_{\odot}\right)$ only slightly exceeds the mass that makes up the hot gas of the entire superbubble $\left(1.8 \times 10^{3} M_{\odot}\right)$. However, this extra mass will be injected in a small portion of the interior (see below). In addition, champagne flows will dump kinetic energy in the form of turbulent motion into the hot cavity. In principle, the injected turbulent energy is more than the thermal energy of the hot gas provided by the SN that led to the formation of the Barnard's Loop bubble (Table 3). However, dissipation of this turbulence will lead to temperatures of only $\sim 10^{4} \mathrm{~K}$ (for a flow speed of $\sim 30 \mathrm{~km} \mathrm{~s}^{-1}$; Table 3; Tielens 2005). The cooling function peaks at these temperatures (Dalgarno \& McCray 1972) such that this excess energy will be mainly radiated away. Nevertheless, adding and mixing in the champagne gas will mass-load and cool the hot superbubble interior gas. In addition, thermal evaporation from the dozens of clouds that are submersed in the hot gas of the superbubble, most notably the OMCs, will contribute to the mass loading of the hot gas. It is difficult to constrain the relative contributions to the mass loading of the hot gas through thermal evaporation and photoevaporation, as the thermal evaporation rate is a steep function of temperature ( $\propto T^{5 / 2}$; Cowie \& McKee 1977), while photoevaporation rates depend on the ionizing luminosity and main-sequence lifetime of the ionizing star (e.g., Bodenheimer et al. 1979; Whitworth 1979). Nonetheless, we can infer that the combined effects of the mass-loading mechanisms have introduced some $6.7 \times 10^{3} M_{\odot}$ of material into the superbubble cavity over the past $3 \times 10^{5}$ yr given by the mass and age of the $\mathrm{H} \alpha$ shell associated with the Barnard's Loop bubble that acts to cleanse the interior of the superbubble close to the OMCs. These numbers imply an average evaporation rate (or cloud destruction rate) of $\sim 1 \times 10^{-2} M_{\odot}$ $\mathrm{yr}^{-1}$, which is an order of magnitude above the current photoerosion rate in the Orion Nebula, if we use a total ionized gas mass of $20 M_{\odot}$ (Wilson et al. 1997) and a dynamical lifetime of $1.5 \times 10^{4} \mathrm{yr}$ (O'Dell et al. 2009). Part of this discrepancy may be because the Orion Nebula still resides in an early phase of evolution and the region has not fully broken out of its natal cloud. Nonetheless, mass loading of the superbubble interior increased the density by a factor of $\sim 100$ compared to that expected in the case in which the region within the Barnard's Loop bubble was filled with hot gas of the same characteristics as observed in the superbubble (Table 3). Over time, this will have a rigorous effect on the thermal behavior of the interior gas.

We note that the preshock density derived for the HV gas associated with the Barnard's Loop bubble in the line of sight toward $\zeta$ Ori (Section 3.3) is $3 \times 10^{-3} \mathrm{~cm}^{-3}$, while we have derived an average density of $1 \mathrm{~cm}^{-3}$ for the medium in which the Barnard's Loop bubble has expanded in its 3 $\times 10^{5} \mathrm{yr}$ lifetime. Surely, the mass-loading mechanisms described here concentrate toward the center of star formation, i.e., the OMCs. There are believed to have been 10-20 SN 
explosions within Orion in the past $12 \mathrm{Myr}$ (Bally 2008). With an expansion velocity of $\sim 30 \mathrm{~km} \mathrm{~s}^{-1}$ for the gas introduced through photoablation and champagne flows (Table 3), an SN rate of $1-1.5 \mathrm{Myr}^{-1}$ will not allow the injected mass to disperse and fill the entire volume of the superbubble before being swept up by a subsequent SNR. Thus, the increased densities due to mass loading from the OMCs will be mainly concentrated within $30-45 \mathrm{pc}$ surrounding the $\mathrm{OB}$ association (consistent with the radius of the Barnard's Loop bubble), whereas the surrounding medium will have a much lower density. The low temperature in the eastern part of the superbubble between Barnard's Loop and region 1 (Section 4.1.3) may also be caused by the effects of mass loading. This would imply that other "routes" of mass loading affect the density and temperature structure of this region, such as enclosed clouds toward the Galactic plane, the straight evaporation of the superbubble wall, or SNRs that dissipate before reaching the superbubble wall (see below). However, as we have not been able to constrain the density structure of this region, the importance of mass loading remains unclear.

In contrast to the shell of the Barnard's Loop bubble that is kept photoionized by Orion OB1 (Section 3.2.2), the neutral shells surrounding $\lambda$ Ori and GS206-17+13 have had the chance to cool radiatively and collapse to form dense media. While they may add to the mass loading of the superbubble interior through thermal evaporation, their slow expansion renders them unimportant compared to the total energetics of the superbubble (Table 3). Still, these dense shells may be the site for a new generation of stars as they are prone to gravitational instabilities (Zavagno et al. 2007; Pomarès et al. 2009; Martins et al. 2010).

The SNR associated with the Barnard's Loop bubble has exploded $\sim 3 \times 10^{5} \mathrm{yr}$ ago and currently expands at $100 \mathrm{~km} \mathrm{~s}^{-1}$, implying that it is well in the momentum-conserving snowplow phase (e.g., Tielens 2005). The postshock gas contains densities $\left(n=3.4 \mathrm{~cm}^{-3}\right.$; Section 3.1$)$ and temperatures $(T=6000 \mathrm{~K}$; Heiles et al. 2000; Madsen et al. 2006; O'Dell et al. 2011) that would allow the gas to cool rapidly $\left(\tau_{\text {cool }} \sim\right.$ $k T / n \Lambda(T) \sim 4 \times 10^{4} \mathrm{yr}$, where $\Lambda(T) \approx 6 \times 10^{-25} \mathrm{erg} \mathrm{cm}^{3} \mathrm{~s}^{-1}$; Dalgarno \& McCray 1972). However, Barnard's Loop is kept photoionized by Orion OB1 (Section 3.2.2), prohibiting the cooling and collapse of the gas to form a dense neutral shell. In this case, the shock wave will continue to gather mass until it becomes subsonic, from which point it will dissipate and pressurize the interior, thus effectively displacing material from the origin of the blast wave toward the outskirts of the superbubble interior.

To get a feeling for how far the displacement of material will reach, we can compare radii and expansion velocities of the Barnard's Loop bubble $\left(r=35 \mathrm{pc}, v_{\exp }=100 \mathrm{~km} \mathrm{~s}^{-1}\right)$ and the superbubble $\left(r=160 \mathrm{pc}, v_{\exp }=40 \mathrm{~km} \mathrm{~s}^{-1}\right)$ to estimate that the Barnard's Loop bubble will catch up with the outer wall within $\sim 2 \mathrm{Myr}$ if both structures decelerate at the same rate (in the radiative expansion phase, the velocity of the SNR decelerates with time as $\propto t^{-5 / 7}$; Tielens 2005). At this moment, the isothermal sound speed in the outer regions of the superbubble toward the east is $\sim 10 \mathrm{~km} \mathrm{~s}^{-1}$ (for $T \sim 10^{4} \mathrm{~K}$; Section 4.1.3). If one assumes the lifetime of Barnard's Loop at $3 \times 10^{5} \mathrm{yr}$ and adopts the time-velocity relation of the SNR above, the adiabatic expansion phase $\left(v_{\exp } \gtrsim 250 \mathrm{~km} \mathrm{~s}^{-1}\right)$ ended just below $10^{5} \mathrm{yr}$, and it will take another $\sim 7 \mathrm{Myr}$ to reach $10 \mathrm{~km} \mathrm{~s}^{-1}$ if the density ahead of the SNR is homeogeneous. This timescale far exceeds that derived for the Barnard's Loop bubble to catch up with the superbubble wall. Thus, Barnard's Loop will be able to cross the superbubble radius and reach the outer wall, where the shock wave will eventually thermalize and the swept-up material will transfer its mass and momentum by condensing onto the superbubble wall (Mac Low \& McCray 1988). The outer superbubble wall may therefore be accelerated as various interior shells such as Barnard's loop catch up to it. In between such pulses, it could be coasting and slowing down as it sweeps up surrounding gas in a momentum-conserving interaction, or it may be dissolving in case it moves subsonically (Section 4.1.3).

We note that densities in the outer parts of the superbubble are smaller compared to the mass-loaded region close to the $\mathrm{OB}$ assocation $\left(\sim 1 \mathrm{~cm}^{-3}\right.$; see above $)$. In this case, the resulting pressure drop will accelerate the interior SNR once again when it reaches the edge of the mass-loading region, which already seems to be the case for the Barnard's Loop bubble, given the preshock density of $3 \times 10^{-3} \mathrm{~cm}^{-3}$ in the line of sight toward $\zeta$ Ori (Welty et al. 2002). Indeed, toward the west side of the superbubble, the temperature of the interior is much higher and the SNR will accelerate, but it may go subsonic and dissolve earlier compared to the east side of the bubble: this may be at the root of the faintness of the $\mathrm{H} \alpha$-emitting shell in this direction (Section 4.1.3). Toward the east, the density gradient could be smaller because of the lower temperature that may be accompanied by an increase in density, and the amount of acceleration will decrease accordingly.

If 10-20 SN explosions occurred in the Orion region in the past $12 \mathrm{Myr}$ (Bally 2008), and each SNR adds $\sim 7 \times 10^{3} M_{\odot}$ of material to the superbubble wall (Table 3), this will amount to $(0.7-1.4) \times 10^{5} M_{\odot}$ and account for a significant fraction $(20 \%-40 \%)$ of its total mass. Thus, the mass of the superbubble wall without the addition from SNRs would account for $(1.9-2.6) \times 10^{5} M_{\odot}$. With a radius of $160 \mathrm{pc}$, we can estimate the average ISM density into which the superbubble has expanded at $n \sim 0.15-0.2 \mathrm{~cm}^{-3}$, consistent with the WNM within one or two scale heights of the Galactic plane. Finally, as the timescale between successive $\mathrm{SNe}$ is of the same order as the timescale the Barnard's Loop bubble to catch up with the outer wall, it may not be necessary to expect multiple layers of expanding shells between the Orion $\mathrm{OB}$ association and the superbubble wall.

\subsubsection{Efficiency of Star Formation in the Orion Molecular Clouds}

Given a total mass of $\sim 2 \times 10^{5} M_{\odot}$ for the OMCs combined, an SN rate of 1-1.5 $\mathrm{Myr}^{-1}$ (Bally 2008), and swept-up mass of $\sim 7 \times 10^{3} M_{\odot}$ per SNR (the mass of the $\mathrm{H} \alpha$-emitting part of the Barnard's Loop bubble), we estimate that at this rate the reservoir of cloud material will be depleted within 20-30 Myr through thermal evaporation and erosion from champagne flows alone, similar to calculated cloud lifetimes from Williams $\&$ McKee (1997). Thus, material is effectively removed from the molecular clouds and incorporated into expanding shells that might form stars of their own. Furthermore, the molecular clouds will be disrupted by each SN blast wave that marks the end of a cycle of star formation. All of these effects should be considered if one attempts to simulate the lifecycle of molecular clouds and the efficiency of star formation, which is well known to be limited by various forms of stellar feedback (for a recent review, see Krumholz et al. 2014, p. 243). 
The gradual erosion of the OMCs puts a firm upper limit on the lifetime of the molecular clouds. Over the past 10-15 Myr, some $\sim 10^{5} M_{\odot}$ has already been lost from the OMCs to the superbubble wall, which implies that the original molecular reservoir (the "proto-Orion" cloud) contained some $\sim 3 \times 10^{5}$ $M_{\odot}$ (Table 3). At present, the major subgroups of the Orion OB association (Blaauw 1964; Brown et al. 1994) constitute some $6.9 \times 10^{3} M_{\odot}$ of stellar mass. The ages of the subgroups imply that many stars have already exploded as SNe (Section 4.3; Bally 2008), and we estimate from the adopted IMF that about $20 \%$ of the initial mass of the 1a, 1b, and 1c subgroups has been lost through SN explosions, increasing the total mass that has been incorporated into stars over the past $10-15 \mathrm{Myr}$ toward $\sim 7.4 \times 10^{3} M_{\odot}$. With a remaining cloud lifetime of the OMCs of 20-30 Myr, at the current rate, some $\sim 2.2 \times 10^{4} \mathrm{M}_{\odot}$, or $7.5 \%$ of the molecular mass, can be converted into stars over the lifetime of the clouds, consistent with predictions by, e.g., Williams \& McKee (1997). However, it is unclear whether star formation will proceed similarly as stellar feedback may trigger star formation and disrupt the clouds, while the molecular reservoir steadily diminishes (see, e.g., Evans et al. 2009; Hopkins et al. 2011; Krumholz et al. 2014, p. 243 for more thorough discussions regarding the mechanisms that involve star formation rates and molecular cloud lifetimes).

\subsubsection{Summary: The Rejuvenation of the Orion-Eridanus Superbubble}

We have argued that appreciable amounts of mass will be removed from the molecular clouds and be mass-loaded into the interior of the superbubble through destructive champagne flows and thermal evaporation of clouds embedded in the hot gas of the superbubble interior. Explosive feedback from stellar winds and, in particular, $\mathrm{SNe}$ take care of the subsequent transportation of the mass-loaded material toward the outskirts of the superbubble through the formation of expanding nested shells. For the Barnard's Loop bubble toward the east, photoionization by Orion OB1 does not allow it to radiatively cool and collapse, and it will plaster the mass and add momentum to the superbubble wall that will continue to drive the expansion of the superbubble. In case it would go subsonic before reaching the outer wall, the shell would dissolve, thermalize, and pressurize the interior. This cycle repeats as long as there is ongoing star formation and OB stars can maintain (the photoionization of) the cleansing shells that deposit the mass toward the outer regions.

\subsection{Dust Processing in Superbubbles}

An interesting consequence of the constant mass loading of the superbubble is that each SN explosion within the superbubble cavity will interact with a significant amount of "fresh" interstellar gas that carries along dust in its wake through entrainment. The efficiency of dust entrainment in photoevaporation flows was investigated by Ochsendorf \& Tielens (2015) for the IC 434 champagne flow, revealing that the dustto-gas ratio within the flow is similar to that seen in the diffuse ISM $(\sim 0.01)$. Thus, each single SNR from Orion OB1 will encounter a new reservoir of gas and dust within the superbubble cavity, introduced by champagne flows from the $\mathrm{H}$ iI regions and from photoevaporation flows of the thermally evaporating clouds. This finding contrasts with that of McKee (1989), who previously estimated the timescale for SNR shocks to destroy a typical dust grain within the ISM. McKee (1989) assumed that only the first SN within an OB association would destroy an amount of dust equivalent to that located in an ISM volume containing $\sim 1300 M_{\odot}$ of gas. Following SNe from the $\mathrm{OB}$ association would hammer surrounding gas already cleansed of its dust component. Following McKee (1989), the effective SN rate is equal to

$$
\tau_{\mathrm{SN}}^{-1}=\left(q_{\mathrm{I}} f_{\mathrm{I}}+\left[f_{\mathrm{II}, \mathrm{OB}} q_{\mathrm{II}, \mathrm{OB}}+f_{\text {II,field }} q_{\mathrm{II}, \text { field }}\right] f_{\mathrm{II}}\right) \kappa_{\mathrm{SN}} \text { years. }
$$

Here $q_{\mathrm{I}}$ is the correlation factor of the Type Ia SNe $(\sim 0.38$; Heiles 1987), relating the location of the $\mathrm{SN}$ to their position above the disk of the Galaxy (i.e., it measures the effective interaction of the $\mathrm{SNe}$ with the amount of gas in their environment), and $f_{\mathrm{I}}$ is the fraction of $\mathrm{SNe}$ Ia $(\sim 0.5$; Narayan 1987). Similarly, $f_{\mathrm{II}}\left(=1-f_{\mathrm{I}}\right)$ measures the fraction of Type II SNe, part of which occur in OB associations, described by $f_{\text {II, OB }}(\sim 0.75$; see Zinnecker \& Yorke 2007, and references therein), and in the field, denoted with $f_{\text {II,field }}(=1-$ $\left.f_{\mathrm{II}, \mathrm{OB}}\right)$, originating from runaways and isolated stars with correlation factor $q_{\text {II,field }}(\sim 0.6$; using values for the scale height of the WNM by Heiles 1987). Finally, $\kappa_{\mathrm{SN}}$ is the intrinsic $\mathrm{SN}$ rate in the Galaxy $\left(\sim 2 \times 10^{-2} \mathrm{yr}^{-1}\right.$; Diehl et al. 2006).

Under the assumption that superbubbles do not replenish their dust content after the first SNR, McKee (1989) arrived at a correlation factor of $q_{\mathrm{II}, \mathrm{OB}}=0.1$ and, together with a rather low value for the fraction of SNe II in OB associations, $f_{\text {II, OB }}$ $=0.5$, derived an effective $\mathrm{SN}$ rate of $\tau_{\mathrm{SN}}=125 \mathrm{yr}$. In this work, we have shown that dust entrainment accompanying mass loading of the superbubble interior replenishes the dust content inside the superbubble after each SNR from the OB association, i.e., $q_{\mathrm{II}, \mathrm{OB}}=1$. In addition, we use the more recently derived fraction of $\mathrm{OB}$ stars that are located in $\mathrm{OB}$ associations from Zinnecker \& Yorke (2007), $f_{\text {II,OB }}=0.75$. These changes lead to a shorter effective $\mathrm{SN}$ rate of $\tau_{\mathrm{SN}}$ $=90 \mathrm{yr}$, implying that the SNRs within superbubbles destroy dust more efficiently and, subsequently, the dust lifetime against SNR shocks must be revised downward by $30 \%$.

The processing of dust inside the superbubble can also be probed directly from depletion studies. Welty et al. (2002) noted that in the HV and IV gas toward $\zeta$ Ori, the gas-phase abundances of $\mathrm{Al}, \mathrm{Si}$, and $\mathrm{Fe}$ are slightly elevated compared to that observed in warm, diffuse clouds. However, the carbon abundance is significantly raised in the HV gas to near-solar abundances. For comparison, depletion studies of the cold neutral medium and the WNM show a pattern where a thin coating on interstellar dust gets sputtered and reaccreted, as the grains cycle from clouds to the diffuse phase of the ISM (Tielens 1998, 2013, p. 499). While in the WNM, the reservoir of gas and dust typically encounters a $100 \mathrm{~km} \mathrm{~s}^{-1}$ passing shock, destroying some $10 \%-30 \%$ of the silicate volume (Jones et al. 1996). However, these shock velocities would only destroy $15 \%$ of the carbonaceous dust and lead to minor variations in the carbon depletion, as $\sim 50 \%$ of the carbon is in the gas phase (Cardelli et al. 1996; Sofia et al. 2004). Therefore, instead of passing shocks, we attribute the enhanced carbon abundance to sputtering of the grains inside the hot gas of the Orion-Eridanus superbubble. The sputtering timescale $\tau_{\text {sput }}$ (Tielens et al. 1994) in the X-ray-emitting gas $\left(T \sim 10^{6} \mathrm{~K}\right.$, $n \sim 0.01 \mathrm{~cm}^{-3}$ ) implies that large $0.1 \mu \mathrm{m}$ silicate grains are 
hardly affected $\left(\tau_{\text {sput }}>20 \mathrm{Myr}\right)$, yet small $\sim 10 \AA$ (carbonaceous) grains are quickly eroded $\left(\tau_{\text {sput }}<1 \mathrm{Myr}\right)$, releasing the carbon into the gas phase. We argue that this may be a general characteristic for dust that is processed inside superbubbles. Indeed, dust entering the heliosphere also shows a significant increase of gas-phase carbon, implying that the grains are sputtered within the hot gas of the Local Bubble (Frisch \& Slavin 2013).

\section{SUMMARY}

The general picture that emerges from the multitude of data is that the Orion-Eridanus superbubble is larger and more complex than previously thought, consisting of a series of nested shells, which we depict in Figure 6. With the data currently at hand, we have discussed the following structures, ordered in size from bottom up.

1. The youngest shell surrounds the ONC located in Orion A (age $<1$ Myr; Bally 2008).

2. Next, there is the $2^{\circ}-4^{\circ} \mathrm{HI}$ and dust shell GS206-17+13, approximately centered on the $\sigma$ Ori cluster, but likely blown by stellar winds from the Belt stars (age $\sim 5 \mathrm{Myr}$; Ochsendorf \& Tielens 2015).

3. Barnard's Loop is part of a complete bubble structure not related to the outer edge of the Orion-Eridanus superbubble. It is instead associated with an SNR (age $~ 3$ $\times 10^{5} \mathrm{yr}$ ) and is connected with HV gas ("Orion's Cloak") detected in absorption studies (Cowie et al. 1979; Welty et al. 2002). All of the previous shells are projected in the interior of this young $7^{\circ}$ radius bubble, which expands at high velocity ( $v_{\exp } \sim$ $100 \mathrm{~km} \mathrm{~s}^{-1}$ ).

4. The Barnard's Loop bubble and the $\lambda$ Ori bubble ( $\sim 5 \mathrm{Myr}$; Bally 2008) expand in the interior of the $\sim 45 \times 45^{\circ}$ shell that is related to IV gas from the aforementioned absorption studies and created by the collective effects of Orion's stars: the Orion-Eridanus superbubble (age $\sim 5-10 \mathrm{Myr}$ ).

As we can recognize several distinct subgroups and welldefined clusters, star formation in the Orion-Eridanus region must have been highly episodic over the past $10-15$ Myr. It may therefore not be surprising that the Orion-Eridanus superbubble consists of a set of nested shells. Each "burst" of star formation may have produced a subgroup consisting of several clusters that disrupted parts of the preexisting molecular cloud. The formation of each subgroup and the resulting UV radiation can ionize large amounts from the dense reservoir of molecular gas in the region that, together with thermal evaporation of the molecular clouds, constantly mass-load and cool the hot gas inside the superbubble cavity. Stellar winds and $\mathrm{SNe}$ accelerate, sweep up, and compress these "poisoned" plasmas in an episodic fashion to form nested shells within the Orion-Eridanus superbubble, such as the Barnard's Loop bubble, the $\lambda$ Ori bubble, the GS206-17+13 shell, and the Orion Nebula (see Figure 6). The shells may cool, collapse, fragment, and be incorporated in a next generation of stars. However, for the Barnard's Loop bubble, this shell cooling is inhibited by photoionization from the $\mathrm{OB}$ association. We have noted that in its turn, the Orion-Eridanus superbubble has previously been associated with the ancient supershell known as Lindblad's ring, which may have cooled and collapsed to form the Gould's Belt of stars that includes the Orion OB association, extending the hierarchy of nested shells and bubbles toward greater sizes and into history. The continuous replenishment of dust in the superbubble cavity through entrainment in ionized flows has led us to conclude that dust processing from interior SNRs is more efficient than previously thought. To conclude, the cycle of mass loading, cleansing, and star formation ceases when feedback has disrupted the molecular reservoir, from which the superbubble will disappear and merge with the ISM.

The authors thank the anonymous referee for detailed comments that significantly increased the quality of this paper. Studies of interstellar dust and chemistry at Leiden Observatory are supported through advanced ERC grant 246976 from the European Research Council; through a grant by the Dutch Science Agency, NWO, as part of the Dutch Astrochemistry Network; and through the Spinoza premie from the Dutch Science Agency, NWO. J.B. was supported in part by National Science Foundatoin (NSF) grant AST-1009847.

\section{REFERENCES}

Alves, J., \& Bouy, H. 2012, A\&A, 547, A97

Bally, J. 2008, in Handbook of Star Forming Regions, ed. B. Reipurth (San Francisco, CA: ASP), 459

Bally, J., Langer, W. D., Stark, A. A., \& Wilson, R. W. 1987, ApJL, 312, L45 Bally, J., Walawender, J., Reipurth, B., \& Megeath, S. T. 2009, AJ, 137, 3843 Barnard, E. E. 1894, PA, 2, 151

Barrado y Navascués, D., Stauffer, J. R., Bouvier, J., Jayawardhana, R., \& Cuillandre, J.-C. 2004, ApJ, 610, 1064

Basu, S., Johnstone, D., \& Martin, P. G. 1999, ApJ, 516, 843

Bedijn, P. J., \& Tenorio-Tagle, G. 1981, A\&A, 98, 85

Blaauw, A. 1964, ARA\&A, 2, 213

Bodenheimer, P., Tenorio-Tagle, G., \& Yorke, H. W. 1979, ApJ, 233, 85

Bohlin, R. C., Savage, B. D., \& Drake, J. F. 1978, ApJ, 224, 132

Bouy, H., Alves, J., Bertin, E., Sarro, L. M., \& Barrado, D. 2014, A\&A, 564, A29

Brown, A. G. A., de Geus, E. J., \& de Zeeuw, P. T. 1994, A\&A, 289, 101

Brown, A. G. A., Hartmann, D., \& Burton, W. B. 1995, A\&A, 300, 903

Burrows, D. N., Singh, K. P., Nousek, J. A., Garmire, G. P., \& Good, J. 1993, ApJ, 406, 97

Cardelli, J. A., Meyer, D. M., Jura, M., \& Savage, B. D. 1996, ApJ, 467, 334 Chevalier, R. A. 1974, ApJ, 188, 501

Chu, Y.-H. 2008, in IAU Symp. 250, Massive Stars as Cosmic Engines, ed. F. Bresolin, P. A. Crowther \& J. Puls (Paris: IAU), 341

Comeron, F., Rieke, G. H., \& Rieke, M. J. 1996, ApJ, 473, 294

Coşkunoğlu, B., Ak, S., Bilir, S., et al. 2011, MNRAS, 412, 1237

Cowie, L. L., \& McKee, C. F. 1977, ApJ, 211, 135

Cowie, L. L., McKee, C. F., \& Ostriker, J. P. 1981, ApJ, 247, 908

Cowie, L. L., Songaila, A., \& York, D. G. 1979, ApJ, 230, 469

Cowie, L. L., \& York, D. G. 1978, ApJ, 220, 129

Cox, N. L. J., Kerschbaum, F., van Marle, A.-J., et al. 2012, A\&A, 537, A35

Dalgarno, A., \& McCray, R. A. 1972, ARA\&A, 10, 375

de Bruijne, J. H. J. 2012, Ap\&SS, 341, 31

Dennison, B., Simonetti, J. H., \& Topasna, G. A. 1998, PASA, 15, 147

de Zeeuw, P. T., Hoogerwerf, R., de Bruijne, J. H. J., Brown, A. G. A., \& Blaauw, A. 1999, AJ, 117, 354

Diehl, R., Halloin, H., Kretschmer, K., et al. 2006, Natur, 439, 45

Dolan, C. J., \& Mathieu, R. D. 1999, AJ, 118, 2409

Dolan, C. J., \& Mathieu, R. D. 2001, AJ, 121, 2124

Draine, B. T. 2011, Physics of the Interstellar and Intergalactic Medium (Princeton: Princeton Univ. Press)

Ehlerová, S., \& Palouš, J. 2005, A\&A, 437, 101

Elmegreen, B. G., \& Lada, C. J. 1977, ApJ, 214, 725

Evans, N. J., II, Dunham, M. M., Jørgensen, J. K., et al. 2009, ApJS, 181, 321 Finkbeiner, D. P. 2003, ApJS, 146, 407

Frisch, P. C., \& Slavin, J. D. 2013, EP\&S, 65, 175

Gaustad, J. E., McCullough, P. R., Rosing, W., \& van Buren, D. 2001, PASP, 113,1326

Gontcharov, G. A. 2006, AstL, 32, 759

Güdel, M., Briggs, K. R., Montmerle, T., et al. 2008, Sci, 319, 309

Habing, H. J. 1968, BAN, 19, 421 
Haffner, L. M., Reynolds, R. J., Tufte, S. L., et al. 2003, ApJS, 149, 405 Hartquist, T. W., Dyson, J. E., Pettini, M., \& Smith, L. J. 1986, MNRAS, 221,715

Heiles, C. 1987, ApJ, 315, 555

Heiles, C., Haffner, L. M., \& Reynolds, R. J. 1999, in ASP Conf. Ser. 168, New Perspectives on the Interstellar Medium, ed. A. R. Taylor, T. L. Landecker \& G. Joncas (San Francisco, CA: ASP), 211

Heiles, C., Haffner, L. M., Reynolds, R. J., \& Tufte, S. L. 2000, ApJ, 536, 335

Hernández, J., Morales-Calderon, M., Calvet, N., et al. 2010, ApJ, 722, 1226

Heyer, M. H., Morgan, J., Schloerb, F. P., Snell, R. L., \& Goldsmith, P. F. 1992, ApJL, 395, L99

Hillenbrand, L. A. 1997, AJ, 113, 1733

Hopkins, P. F., Quataert, E., \& Murray, N. 2011, MNRAS, 417, 950

Huang, J.-S., Songaila, A., Cowie, L. L., \& Jenkins, E. B. 1995, ApJ, 450, 163

Jones, A. P., Tielens, A. G. G. M., \& Hollenbach, D. J. 1996, ApJ, 469, 740

Kalberla, P. M. W., Burton, W. B., Hartmann, D., et al. 2005, A\&A, 440, 775

Kroupa, P. 2001, MNRAS, 322, 231

Krumholz, M. R., Bate, M. R., Arce, H. G., et al. 2014, in Protostars and Planets VI, ed. H. Beuther, R. S. Klessen, C. P. Dullemond, \& T. Henning (Tucson, AZ: Univ. Arizona Press), 243

Lang, W. J., \& Masheder, M. R. W. 1998, PASA, 15, 70

Mac Low, M.-M., \& McCray, R. 1988, ApJ, 324, 776

Maddalena, R. J., \& Morris, M. 1987, ApJ, 323, 179

Madsen, G. J., Reynolds, R. J., \& Haffner, L. M. 2006, ApJ, 652, 401

Martins, F., Pomarès, M., Deharveng, L., Zavagno, A., \& Bouret, J. C. 2010 , A\&A, 510, A32

Martins, F., Schaerer, D., \& Hillier, D. J. 2005, A\&A, 436, 1049

McKee, C. 1989, in IAU Symp. 135, Interstellar Dust, ed. L. J. Allamandola \& A. G. G. M. Tielens (Dordrecht: Kluwer), 431

McKee, C. F., \& Ostriker, J. P. 1977, ApJ, 218, 148

Menten, K. M., Reid, M. J., Forbrich, J., \& Brunthaler, A. 2007, A\&A, 474,515

Morrison, R., \& McCammon, D. 1983, ApJ, 270, 119

Narayan, R. 1987, ApJ, 319, 162

Ochsendorf, B. B., Cox, N. L. J., Krijt, S., et al. 2014a, A\&A, 563, A65

Ochsendorf, B. B., \& Tielens, A. G. G. M. 2015, A\&A, 576, A2

Ochsendorf, B. B., Verdolini, S., Cox, N. L. J., et al. 2014b, A\&A, 566, A75

O'Dell, C. R. 2001, ARA\&A, 39, 99

O’Dell, C. R., Ferland, G. J., Porter, R. L., \& van Hoof, P. A. M. 2011, ApJ, 733, 9

O’Dell, C. R., Henney, W. J., Abel, N. P., Ferland, G. J., \& Arthur, S. J. 2009, AJ, 137, 367

Osterbrock, D. E., \& Ferland, G. J. 2006, Astrophysics of Gaseous Nebulae and Active Galactic Nuclei (Sausalito, CA: University Science Books)

Paladini, R., Umana, G., Veneziani, M., et al. 2012, ApJ, 760, 149

Perryman, M. A. C., de Boer, K. S., Gilmore, G., et al. 2001, A\&A, 369, 339

Perryman, M. A. C., Lindegren, L., Kovalevsky, J., et al. 1997, A\&A, 323, L49

Planck Collaboration, Abergel, A., Ade, P. A. R., et al. 2014a, A\&A, 571, A11

Planck Collaboration, Ade, P. A. R., Aghanim, N., et al. 2011, A\&A, 536, A1
Planck Collaboration, Ade, P. A. R., Aghanim, N., et al. 2014b, arXiv: 1409.2495

Planck Collaboration, Ade, P. A. R., Aghanim, N., et al. 2014c, A\&A, 571, A1 Pomarès, M., Zavagno, A., Deharveng, L., et al. 2009, A\&A, 494, 987

Pon, A., Johnstone, D., Bally, J., \& Heiles, C. 2014a, MNRAS, 441, 1095

Pon, A., Johnstone, D., Bally, J., \& Heiles, C. 2014b, MNRAS, 444, 3657

Reich, W. 1978, A\&A, 64, 407

Reynolds, R. J., \& Ogden, P. M. 1979, ApJ, 229, 942

Sandstrom, K. M., Peek, J. E. G., Bower, G. C., Bolatto, A. D., \& Plambeck, R. L. 2007, ApJ, 667, 1161

Schlafly, E. F., Green, G., Finkbeiner, D. P., et al. 2014, ApJ, 786, 29

Schlafly, E. F., Green, G., Finkbeiner, D. P., et al. 2015, ApJ, 799, 116

Sherry, W. H., Walter, F. M., \& Wolk, S. J. 2004, AJ, 128, 2316

Snowden, S. L., Burrows, D. N., Sanders, W. T., Aschenbach, B., \& Pfeffermann, E. 1995, ApJ, 439, 399

Snowden, S. L., Egger, R., Freyberg, M. J., et al. 1997, ApJ, 485, 125

Sofia, U. J., Lauroesch, J. T., Meyer, D. M., \& Cartledge, S. I. B. 2004, ApJ, 605,272

Spitzer, L. 1978, Physical Processes in the Interstellar Medium (New York: Wiley)

Tenorio-Tagle, G. 1979, A\&A, 71, 59

Tielens, A. G. G. M. 1998, ApJ, 499, 267

Tielens, A. G. G. M. 2005, The Physics and Chemistry of the Interstellar Medium (Cambridge: Cambridge Univ. Press)

Tielens, A. G. G. M. 2013, in PAHs and Dust, ed. T. D. Oswalt \& G. Gilmore (Dordrecht: Springer)

Tielens, A. G. G. M., \& Hollenbach, D. 1985, ApJ, 291, 722

Tielens, A. G. G. M., McKee, C. F., Seab, C. G., \& Hollenbach, D. J. 1994, ApJ, 431, 321

van Buren, D. 1986, ApJ, 306, 538

van der Werf, P. P., Goss, W. M., \& O’Dell, C. R. 2013, ApJ, 762, 101

van Leeuwen, F. 2007, A\&A, 474, 653

Veilleux, S., Cecil, G., \& Bland-Hawthorn, J. 2005, ARA\&A, 43, 769

Wall, W. F., Reach, W. T., Hauser, M. G., et al. 1996, ApJ, 456, 566

Warren, W. H., Jr., \& Hesser, J. E. 1977, ApJS, 34, 115

Weaver, R., McCray, R., Castor, J., Shapiro, P., \& Moore, R. 1977, ApJ, 218, 377

Welty, D. E., Jenkins, E. B., Raymond, J. C., Mallouris, C., \& York, D. G. 2002, ApJ, 579, 304

Wen, Z., \& O'Dell, C. R. 1995, ApJ, 438, 784

Whitworth, A. 1979, MNRAS, 186, 59

Williams, J. P., \& McKee, C. F. 1997, ApJ, 476, 166

Wilson, B. A., Dame, T. M., Masheder, M. R. W., \& Thaddeus, P. 2005, A\&A, 430,523

Wilson, T. L., Filges, L., Codella, C., Reich, W., \& Reich, P. 1997, A\&A, 327, 1177

Wright, E. L., Eisenhardt, P. R. M., Mainzer, A. K., et al. 2010, AJ, 140, 1868

Zavagno, A., Pomarès, M., Deharveng, L., et al. 2007, A\&A, 472, 835

Zinnecker, H., \& Yorke, H. W. 2007, ARA\&A, 45, 481 